\title{
Realistic Solar Heating in Urban Areas: Air Exchange and Street-Canyon Ventilation
}

\author{
N. Nazarian ${ }^{\mathrm{a}, *}$, J. Kleissl ${ }^{\mathrm{a}}$ \\ ${ }^{a}$ Mechanical and Aerospace Engineering, University of California, San Diego, 9500 Gilman Dr. 0411, La Jolla, CA \\ 92093-0411
}

\begin{abstract}
Numerical fluid flow and heat transfer simulations of a three-dimensional idealized urban environment are performed to investigate the effect of realistic non-uniform thermal forcing that is caused by solar insolation and inter-building shadowing. Simulations at different times of day are performed using Large Eddy Simulation (LES) and mean flow and turbulence statistics are investigated as determinants for urban canyon ventilation. The differential surface heating of the building canyon is parameterized using sets of horizontal and vertical Richardson numbers indicating atmospheric instability and solar tilt with respect to the wind direction, respectively. Roof heating, in combination with building walls and ground heating, is shown to be important in the strength and location of the canyon vortex. For example, in case of weak vertical stratification $\left(R i_{v}\right)$ and high horizontal temperature gradient opposing the wind direction above the canyon $\left(R i_{h}\right)$, the combination of roof heating and ground heating decreases the strength of the canyon vortex. The distribution of local convective heat transfer coefficients (CHTC) on building facets are analyzed. Throughout the day, the windward walls exhibit larger CHTCs and leeward heating enhances CHTC from the roof and windward walls. Additionally, the wall heating in the cross-stream canyon (north-south in our case) enhances the air exchange rate from the canyon, especially when the leeward wall is heated.
\end{abstract}

Keywords: Urban heat transfer, Computational Fluid Dynamics, Convective Heat Transfer Coefficients, Street Air Exchange

\section{Introduction}

The world is currently experiencing the largest wave of urbanization in history. According to the 2011 World Urbanization Prospects [1], more than $78 \%$ of the population in developed countries already lives in urban areas and the fraction is expected to increase worldwide. As urbanization progresses, microclimate modifications are aggravated in various ways [2-4] and Urban Heat Island effects become more prevalent [5]. The progressive replacement of natural surfaces by impervious materials and buildings modifies regional morphology and land cover. The urban radiation balance is altered by the reflection and obstruction of solar radiation as well as the reduction of thermal radiation losses due to small sky view factors [5]. Building roughness provides a momentum sink and causes wind sheltering within street canyons. The contrast between the urban and undisturbed climates is further enhanced by the input of anthropogenic heat, moisture and pollutants into the atmosphere by human activities [6]. Therefore, it is essential that we develop more sophisticated and comprehensive methods of urban thermal and flow analysis in order to investigate the combined effect of various urban climate processes.

\footnotetext{
*Corresponding author, Tel.: +1 8586999870

Email addresses: nenazarian@ucsd.edu (N. Nazarian), jkleissl@ucsd.edu (J. Kleissl)
} 


\section{Nomenclature}

\begin{tabular}{|c|c|c|c|}
\hline \multicolumn{2}{|c|}{ Abbreviations } & \multirow{2}{*}{$\begin{array}{l}k \\
L\end{array}$} & \multirow{2}{*}{$\begin{array}{l}\text { Effective thermal conductivity of the ground } \\
\text { Net longwave radiation flux }\end{array}$} \\
\hline \multicolumn{2}{|c|}{ ACH Air Exchange Rate } & & \\
\hline \multicolumn{2}{|r|}{ Building Aspect Ratio, $\mathrm{AR}=H / W$} & $L_{i n c}$ & Incident longwave radiation \\
\hline & Detached Eddy Simulation & $N u$ & Nusselt Number $N u=\frac{h H}{h}$ \\
\hline & Dynamic Smagorinsky-Lilly (model) & $Q_{c}$ & Conduction heat flux $\quad k$ \\
\hline LES & Large Eddy Simulation & Qc & Conduction heat flux \\
\hline \multirow{2}{*}{$\begin{array}{l}\text { RANS } \\
\text { TMY }\end{array}$} & Reynolds-Averaged Navier-Stokes (equations) & $Q_{h}$ & Sensible heat flux \\
\hline & Typical Meteorological Year & $R i$ & Richardson Number \\
\hline \multirow{2}{*}{$\begin{array}{l}\text { WALE } \\
\text { WMLES }\end{array}$} & Wall-Adapting Local Eddy Viscosity (model) & $S$ & Shortwave radiation \\
\hline & Algebraic Wall-Modeled LES (model) & $S_{i j}$ & Strain-Rate tensor \\
\hline \multicolumn{2}{|c|}{ Greek Symbols } & $T_{a}$ & Inlet air temperature \\
\hline$\alpha$ & Surface albedo & $T_{g}$ & Ground surface temperature \\
\hline$\epsilon$ & Surface emissivity & $T_{H}$ & Fluid air temperature at roof level \\
\hline $\begin{array}{l}\sigma \\
\tau_{i j}\end{array}$ & $\begin{array}{l}\text { Stephan-Boltzmann constant } \\
\text { Subgrid-scale stress }\end{array}$ & $T_{L}, T_{W}$ & $\begin{array}{l}\text { Leeward and windward wall temperature, respec- } \\
\text { tively }\end{array}$ \\
\hline & Shear / friction velocity & $T_{s k y}$ & Sky radiation temperature \\
\hline \multicolumn{2}{|c|}{ Roman Symbols } & $U_{b}$ & Average bulk wind velocity at inlet \\
\hline & Plan area of roughness elements & $W$ & 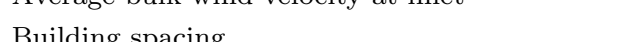 \\
\hline & Total surface area of roughness elements & & Building spacing \\
\hline & Effective heat capacity of the ground & \multicolumn{2}{|c|}{ Operators } \\
\hline \multirow{2}{*}{$\begin{array}{l}F_{g r-w} \\
H\end{array}$} & View Factor from ground to wall & \multirow{2}{*}{$\begin{array}{l}<Q> \\
\bar{Q}\end{array}$} & Spatial average \\
\hline & Building height & & Time average \\
\hline \multirow[t]{2}{*}{$h$} & Convective Heat Transfer Coefficient (CHTC), & $\widehat{Q}$ & Filtered quantity \\
\hline & $h=\frac{Q_{h}}{\left(T-T_{\text {ref }}\right)}$ & $Q^{+}$ & Dimensionless \\
\hline
\end{tabular}

Thermal forcing plays an important role in determining flow patterns and turbulent transport in built environments $[7,8]$. Various surface controls that affect the dynamic thermal and flow field behavior are widely documented in the literature (through numerical modeling or wind tunnel experiments) and are mostly categorized in 4 major factors: 1) building canyon geometry and configuration [9-12], 2) surface thermal properties [13-15], 3) ambient wind speed and direction [15, 16], and 4) distribution and strength of surface heating with respect to the ambient wind [17-22]. When the building facades are heated by solar radiation during the day, buoyancy enhances turbulence [23], and the combination of buoyancy and inertial forces governs the heat and mass removal from the street canyon. For example, when the windward wall is warmer than the air, the upward buoyancy flux opposes the downward mechanical advection in the building canyon, and the flow structure in the building cavity adjusts to these counteracting effects. It is essential to also recognize the three-dimensional (3D) nature of the urban canopy, including the interaction between buildings under non-idealized conditions [24]. For example, variation of solar position and insolation throughout the day result in 3D heterogeneous distribution of thermal forcing on urban facets. Non-uniform, 3D surface heating is not commonly considered in the literature. Since the solar irradiance field is not realistic the flow field within and above the canopy is also not expected to represent real conditions. To address this shortcoming, non-uniform thermal forcing and its 3D effect on urban flow field will be investigated.

Convective heat transfer coefficients (CHTCs) at exterior building surfaces determine turbulent heat fluxes and therefore affect building thermal loads and energy demands, canopy air exchange and pollutant dispersion. Empirical CHTC correlations for external urban surfaces have been evaluated using field measurements [25-30] and wind tunnel experiments [18],[22],[31]. However, due to wind direction variability and complex canopy roughness especially in field experiments, the flow field and therefore the CHTC are very sensitive to the boundary conditions and inconsistencies between measurement campaigns were found [32-34]. Wall resolving Computational Fluid Dynamics (CFD) avoids the need to model Convective Heat Transfer Coefficients at external building surfaces [35-38]. However, the computational cost for simulations at realistic 
Reynolds numbers and over the diurnal cycle is too large. Consequently, simplified correlations are used to calculate or impose the value of CHTC. This results in large discrepancies among some of the widely used building simulation tools as reported by [39] and [40]. The latter suggests $20 \%$ to $40 \%$ difference in energy demands with different choice of Convective Heat Transfer Coefficient. Furthermore, non-uniform and dynamic surface heating and associated changes in the flow field motivate the investigation of spatio-temporal distributions of CHTCs.

The air removal, or the capacity of the street canyon to "ventilate" itself, has a critical role on air quality, city breathability [41] and therefore quality of life of urban dwellers. Urban airflow and pollutant transport has received significant attention and several parameters have been introduced to quantify the ventilation performance. Liu et al ([42] and [43]) considered the roof-level fluctuating vertical velocity as the sole responsible mechanism for air removal and pollutant dilution in the $2 \mathrm{D}$ street canyon. The Air Exchange Rate $(\mathrm{ACH})$ was introduced by Liu et al [44] to represent the volumetric air per unit time that is exchanged from the street canyon to the surrounding atmosphere. ACH was applied to compare the ventilation efficiency of street canyons [45-48]. The differential heating of building surfaces can also influence the exchange of air and pollutants [8]. Yet, numerical investigations on the effect of buoyancy on canyon ventilation have been relatively rare ([20],[46],[49]) and focused on idealized uniform surface heating scenarios. It is expected that non-uniform heating, significantly affects the air exchange rate.

In this paper, numerical fluid flow and heat transfer simulations of a three-dimensional (3D) urban environment are performed using the finite volume solver ANSYS/FLUENT 14.5. CFD simulation of urban microclimate is shown to be a powerful tool that can accurately model the urban thermal environment, as well as providing the possibility for comparative analysis [50]. Several studies demonstrated the importance of including shortwave and longwave radiation on accurately predicting the urban thermal environment and UHI effects in real urban configurations [50-53], however detailed analysis on the effect of thermal forcing on the local (street-scale) flow field is scarce. In this study an idealized compact low-rise geometry is used for generality [54], and the focus is on the detailed study of the determinants of urban thermal and flow environments. Accordingly, the main objective is to analyze the impact of non-uniform thermal forcing caused by varying incidence angles of direct beam shortwave radiation and shading effects between buildings on mean flow, turbulence statistics, temperatures, and canyon ventilation. Our model also extends previous urban analysis of CHTCs through a dynamic coupling of heat transfer and fluid flow in a 3D street-scale urban environment. The differential thermal forcing in the building canyon is parameterized through a new potentially universal dimensionless Richardson number. Additionally, more advanced numerical modeling of turbulent flow (Large Eddy Simulation) is performed to improve the accuracy in the state variables and fluxes compared to RANS.

Section 2 of the present paper explains the simulation setup, including the model description, numerical methods, grid dependency, and test cases. Different turbulence models are compared and results are validated against experimental measurements in Section 3. The relative importance of momentum versus buoyancy forcing is characterized by defining two different Richardson numbers as explained in Section 4.2. Results and discussions are further subdivided in three sections: the impact of three-dimensional nonuniform heating on mean flow field is analyzed in section 4.3, followed by the analysis of CHTC distribution on building walls (Section 4.4), and street canyon ventilation is studied by means of 3D Air Exchange Rate in Section 4.5. Conclusions are presented in section 5.

\section{Methods}

\subsection{Model Description}

The geometry consists of a matrix of $3 \times 3$ equally-spaced buildings with canyon aspect ratio of 1 (AR = Height-to-Width ratio), situated on top of multiple soil layers (Fig. 1). This configuration results in a roughness plan aspect ratio $\lambda_{p}=A_{p} / A_{T}$ of 0.25 , where $A_{p}$ is the plan area of roughness elements relative to the total surface area $A_{T}$. The configuration density is classified as the "compact low-rise" areas in the local climate zone (LCZ) classification by Stewart and Oke [54]. The streamwise, spanwise and vertical coordinates 

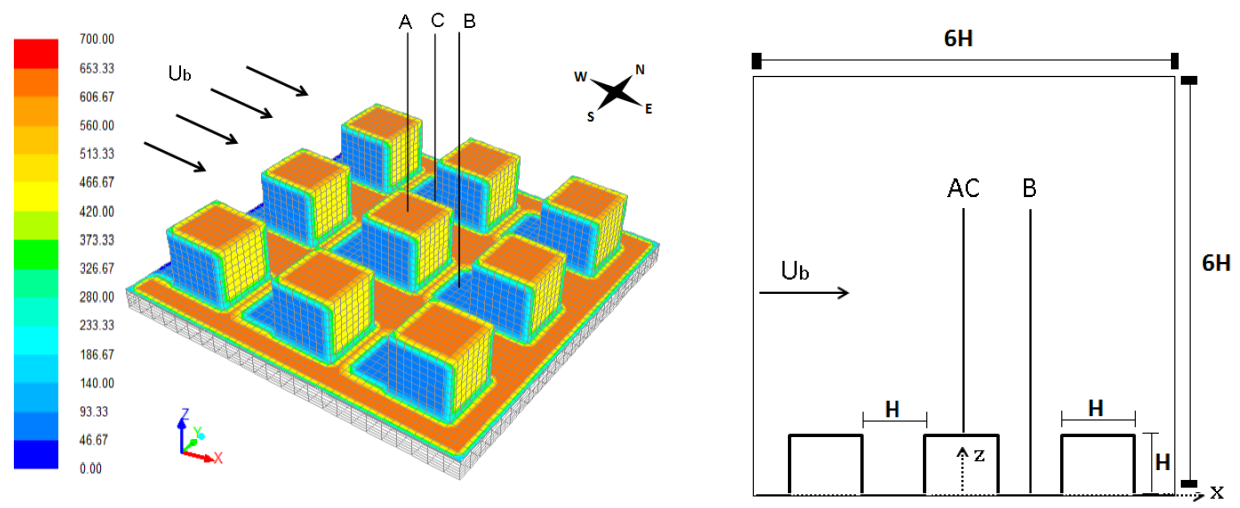

Figure 1: Left: Absorbed solar heat flux $\left(S_{a b s}\right)$ in $\mathrm{W} \mathrm{m}^{-2}$ in the computational domain for ground surface albedo of 0.18 at 1500 LST on June 21st. Right: $X-Z$ view at plane $Y / H=0$ with locations where vertical profiles are analyzed. A: Above center building $X / H=0, Y / H=0$, B: Downwind halfway between buildings $X / H=1, Y / H=0, \mathrm{C}$ : Center-line street canyon $X / H=0, Y / H=1$.

are denoted by $x, y$ and $z$, respectively. To achieve a fully-developed flow field, periodic boundary conditions are used in stream and span-wise directions and a no-shear velocity condition is applied at the domain top $(6 H)$. Nazarian and Kleissl ([15]) performed domain size sensitivity tests with RANS and concluded that larger domain heights do not affect the results, consistent with [55] who suggested a domain height of $4 H$. The dynamic coupling of flow field and heat transfer is taken into account by simultaneously solving the Navier Stokes equations, energy equation, and Discrete Ordinate non-gray radiation model by [56] in the fluid domain. Natural convection from the heated surfaces and buoyancy effects on the flow are considered and the Boussinesq approximation is used for density. The shortwave radiation $(S)$ is calculated using a solar ray tracing algorithm provided in ANSYS/FLUENT [57] that simulates direct solar radiation and shading by buildings. $S$ is applied to the energy equation as a source term for each cell and is the primary driver of non-uniform heating of building and ground surfaces. This approach is more realistic than forcing constant non-uniform heat flux on urban surfaces, as other energy balance components are coupled to $S$. The unsteady conduction heat flux $\left(Q_{c}\right)$ into the thermally massive soil layers is calculated at the ground surface, while the 1D steady heat conduction equation is solved to calculate the external surface temperature of buildings. The thermal properties of soil layers and ground and wall surfaces are as in Nazarian and Kleissl ([15]) and summarized in Appendix A. A constant sky radiation temperature of $255 \mathrm{~K}$ is set at the upper boundary of the computational domain to simulate longwave radiation from clear sky conditions. A constant temperature boundary condition $(295 \mathrm{~K})$ is used at the external solid surfaces of the domain (the base of the deepest soil layer and the internal surface temperature in roofs and walls) as input to the conduction heat flux calculation from the solid layers. While periodic boundary conditions are considered for the velocities, the inflow air temperature $T_{a}$ is forced to be homogeneous and to follow the TMY3 diurnal cycle.

\subsection{Turbulence Modeling and Numerical Methods}

The unstructured finite volume solver ANSYS FLUENT 14.5 is applied with a constant time step of $0.1 \mathrm{~s}$ resulting in average CFL numbers of 0.3 and maximum CFL numbers of 1-1.1 observed locally in areas of high velocity. After a detailed comparison and validation of various turbulence models (section 3), Large Eddy Simulation and Algebraic Wall-Modeled subgrid-scale model (WMLES) is chosen and used with near wall treatment by Werner and Wengle [58] (Section 3). The numerical method is described in detail in Nazarian and Kleissl ([15]) and the key points are restated here. A detailed description of the Large Eddy Simulation model (LES) and Algebraic Wall-Modeled LES Model (WMLES) can be found in Appendix A-C. In the following simulations urban surfaces are considered smooth and the effect of building surface roughness is not included in this study due to the limitation imposed by the ANSYS/FLUENT software that 
does not allow specifying roughness length when WMLES is used combined with the near wall treatment of Werner and Wengle [58].

\subsection{Mesh Configuration and Grid Dependency Analysis}

The 3D Cartesian grids are built with ANSYS 14.5 resolving each cube cavity with 16 cells in all directions. The grid dependency is tested for the computational domain described in $2.1(3 \times 3$ matrix of building with $\mathrm{AR}=1$ and domain height of $6 H$ ). Three structured grid systems as described in Table 1 are used. Computing resources limited the range of grid points for the grid dependency analysis. The $2 \mathrm{H} \times 2 \mathrm{H} \times 6 \mathrm{H}$ subchannel unit of the computational domain (Figure 2) consists of $32 \times 32 \times 52$ control volumes in the flow along the $x, y$ and $z$ directions, respectively for the coarse grid (Base case shown in Table 1). The entire domain contains $9(3 \times 3)$ times the area shown in Figure 2 or 723,600 cells (including 75,000 cells for the soil layers) in the Base case. The high resolution (HR) cases contain $40 \times 40 \times 52$ control volumes and in case HR-NWR, grids are more refined close to the building walls and ground surfaces to achieve smaller dimensionless wall distance $\left(\mathrm{z}^{+}\right)$, which results in better resolution of the energy-containing scales near the wall, but also grid cells with larger aspect-ratio (pencil-like grids) further away from the wall.

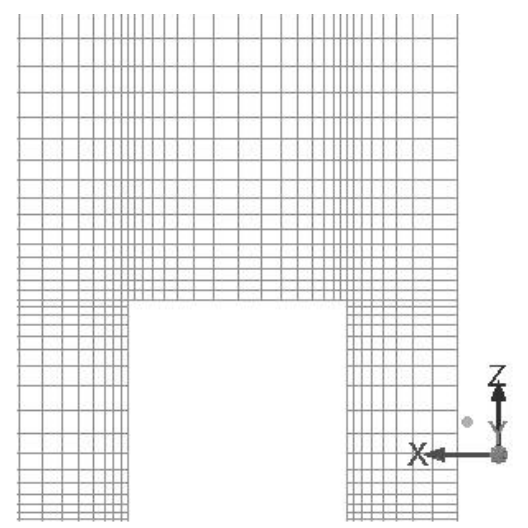

Figure 2: Subsection of the computational grids $(2 \mathrm{Hx} 2 \mathrm{H})$ in the $x-z$ plane around the building canyon for the Base case (Table 1).

\begin{tabular}{l|cccc} 
Case & $N_{x}$ & $N_{y}$ & $N_{z}$ & $\mathbf{z}^{+}$ \\
& $($per & $($per & & \\
& $2 H)$ & $2 H)$ & & \\
\hline Base & 32 & 32 & 52 & 120 \\
HR & 40 & 40 & 52 & 80 \\
HR-NWR & 40 & 40 & 52 & 30
\end{tabular}

Table 1: Numbers of grids within the subchannel unit shown in Fig. 2 and wall distance of the first grid point for the cases simulated in the grid dependency analysis. "HR" stands for "high resolution" and "NWR" refers to near-wall resolution. To obtain the total number of grid points multiply $N_{x}$ and $N_{y}$ by 6 .

Vertical profiles of mean streamwise velocity and streamwise and spanwise velocity variances are compared for different grid resolutions. Downstream of the cube (Location B in Figure 1) refining grids in the horizontal directions does not significantly affect the profile of mean velocity and mean temperature (Base versus HR in Figure 3). The largest Mean Average Percentage Error (MAPE) is observed for the variance of streamwise velocity in Base case with HR at MAPE $=7 \%$. For the HR-NWR case, results away from the wall are affected by the large aspect-ratio in this region, as also observed by Niceno et al 2002 ([18]). Similar independence to grid resolution and near-wall refinement was observed at the locations $\mathrm{A}$ and $\mathrm{C}$ and can be attributed to the robust near-wall approach by Werner and Wengle [58] and the WMLES SGS model used. Therefore the computational grid of the Base case is the best compromise between accuracy and computational cost and used for the following simulations.

\subsection{Simulation Cases During the Diurnal Cycle}

Simulations are conducted for steady-state weather conditions at 06, 08, 10, 12, 14, 16, 18 PST on a clear summer day in San Diego (June 21), and initialized with temperature and wind forcing data using Typical Meteorological Year (TMY3 file for Miramar NAS) data and the solar load model of the corresponding hour. The bulk velocity $U_{b}$ is forced to be the average wind speed in the Typical Meteorological Year (TMY3) data at a representative coastal urban weather station in southern California (San Diego Miramar NAS, 

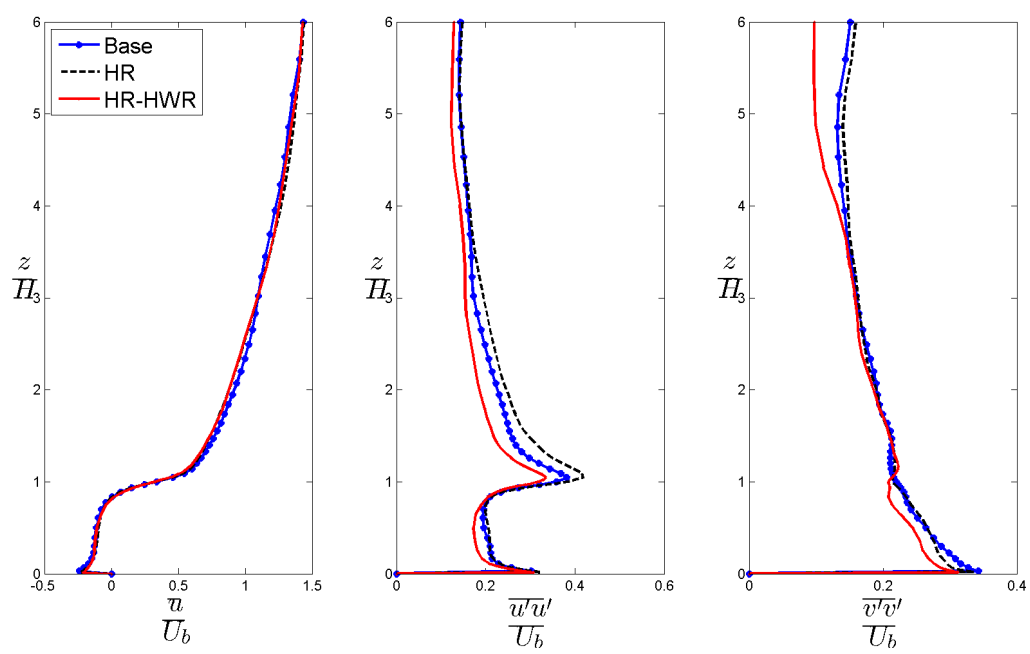

Figure 3: Vertical profiles of mean velocity and velocity variances at Location B (Fig. 1) and different grids (Tab. $1)$.

$\left.32^{\circ} 52^{\prime} \mathrm{N}, 117^{\circ} 09^{\prime} \mathrm{W}\right)$. In the streamwise direction a corresponding mass flow rate is specified and the Reynolds number based on the cube height $R e_{H}=U_{b} H / \nu$ is $5.2 \times 10^{5}$. To confirm that a quasi-steady state $\left(t_{q s}\right)$ was reached before data collection commenced, resolved and normalized resolved turbulent kinetic energy $\left(k^{+}=\frac{k}{u_{\tau}^{2}}\right)$ averaged over the whole domain was monitored as a function of time, where $k$ is the turbulent kinetic energy and $u_{\tau}$ is the friction velocity near ground. The sampling time for computing time averages is set to $1500 \mathrm{~s}$ time interval, which is at least 15 times the Lagrangian integral time scale $\left(t_{L}\right)$ and then output is sampled every 10 time steps $(1 \mathrm{~s})$.

\section{Validation and Turbulence Model comparison}

In order to choose and validate the appropriate turbulence model provided in FLUENT, Detached Eddy Simulations (DES), and Large Eddy Simulations (LES) are compared to an experiment by Meinders [59] and the LES simulation of Niceno et al [18]. The standard Smagorinsky subgrid-scale model was used in [18], and the first grid point was at $z^{+} \approx 1$.

The experimental measurements by Meinders [59] were conducted for a heated cube placed in an equidistantly spaced matrix of wall-mounted cubes in a wind tunnel. The center-to-center distance between cubes are $4 H$, were $H$ is the height of the cubes. The height of the channel is $3.4 H$.

The subchannel unit of dimension $4 H \times 4 H \times 3.4 H$ is chosen as the computational domain and periodic boundary condition are applied for the streamwise and spanwise directions, $x$ and $y$, respectively, consistent with the LES simulation in Niceno et al [18]. However, since only the center cube is heated in Meinders, periodic boundary conditions are not applied for temperature; instead the inlet temperature is fixed to $20^{\circ} \mathrm{C}$. No slip and $20^{\circ} \mathrm{C}$ boundary conditions is also applied for channel floor and domain top. The center cube is heated using a constant-temperature core $\left(75^{\circ} \mathrm{C}\right)$ covered with a thin layer of $0.1 \mathrm{H}$ thickness and thermal conductivity of $0.24 \mathrm{~W} \mathrm{~m}^{-1} \mathrm{~K}^{-1}$. In our simulation the cube thickness is not resolved by grids; instead the 1D steady heat conduction equation is used to calculate the external surface temperature. The computations were performed at a Reynolds number based on the cube height of $R_{H}=U_{b} H / \nu=3,854$, and a corresponding mass flow rate is specified in the stream-wise direction.

The turbulence models are described briefly in sections 2.2 and Appendix B-C. Large Eddy Simulation with two different SGS models and two different Delayed Detached Eddy Simulation models are used in this comparison as summarized in Table 2. Since Heidarzadeh et al. [38] found that WALE (Wall-adapting local 
Table 2: List and acronyms and reference for turbulence models used in the intercomparison.

\begin{tabular}{l|l|l}
\hline \hline LES DS & Large Eddy Simulation with Dynamic Smagorinsky Subgrid-Scale Model & Germano [60] \\
LES WMLES & Large Eddy Simulation with Algebraic Wall Subgrid-Scale Model & Shur et al. [61] \\
DDES SA & Delayed Detached Eddy Simulation based on Spalart-Allmaras & Spalart et al [62] \\
DDES Ke-R & Delayed Detached Eddy Simulation based on $k-\epsilon$ Realizable & -
\end{tabular}

eddy viscosity) models were inferior to DDES models in FLUENT simulations of convective heat transfer over a wall mounted cubes, the WALE model is not included in this comparison. Figures 4 shows the vertical
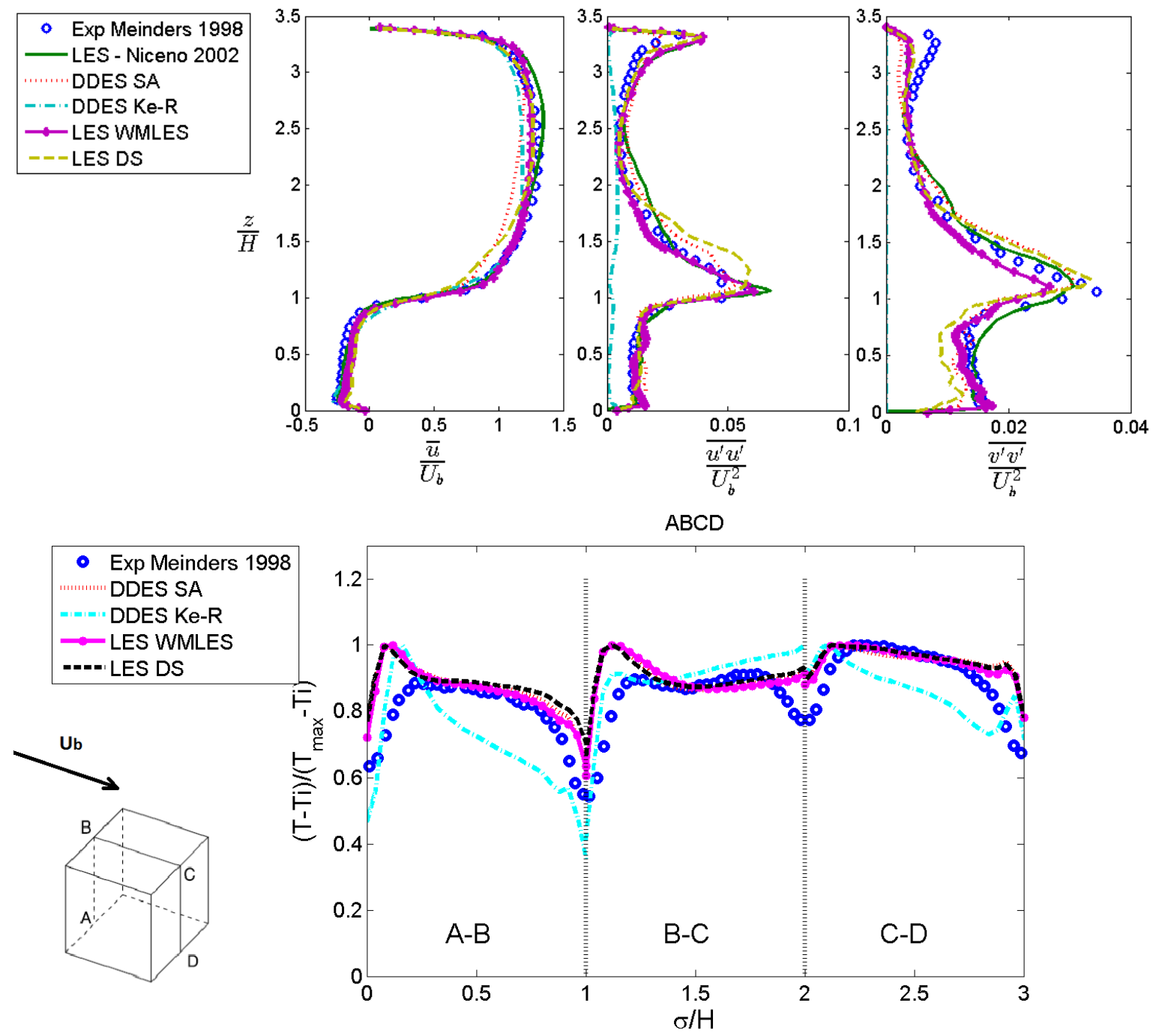

Figure 4: Top: Vertical profiles of mean velocity and velocity variances at Location B (Fig. 1) and different turbulence models (Tab 2). Bottom: Distribution of the normalized surface temperature along the path ABCD.

profile of time-averaged normalized streamwise velocity and the normalized streamwise and spanwise velocity variances in the canyon downwind of the heated building. URANS models are also compared but not included here as they were shown to be inadequate in predicting the velocity fluctuation and other turbulent 
parameters especially in the canyon region. The Algebraic Wall-Modeled Large Eddy Simulation (WMLES) model with near-wall treatment is closest to the experimental data, and this is also observed at the other locations (not shown). The vertical profile of streamwise velocity is captured well, especially in the region close to the wall. On the other hand, the larger vertical grid spacing above the buildings results in larger cell aspect-ratio (pencil-like cells) and negatively influenced the accuracy in this region. Nevertheless the agreement of WMLES results with experimental data for both mean and variance of the streamwise velocity is improved compared to Niceno et al [18], while the spanwise variance is slightly less accurate between $1<z / H>1.5$. The calculated temperature of the building surfaces is also validated following the same methodology and it is concluded (Figure 4) that LES models with Mean Absolute Percentage Error (MAPE) of 7-8.5 percent outperform the DDES model (MAPE of 12.3 percent for DDES Ke-R) on their prediction of urban surface temperatures. The WMLES results are in close agreement with the experimental data in the center sections of the building surfaces, while larger errors exist near the edge of the cubes. The deviation can be attributed to the following reasons. First, a near-wall resolution of $\mathrm{y}+$ of 1 is recommended by Niceno et al. [18] to accurately represent the near surface fields. In our validation study, the y+ ranges from $5-15$, and the maximum occurs at the corners, specifically at the corner of the windward wall. Therefore lower resolution can contribute to the deviation of surface temperature at these locations. Additionally, the temperature boundary condition at the channel floor and domain top is set to be constant in the CFD simulation, while heterogeneity may exist in the experiment as these temperatures are not controlled.

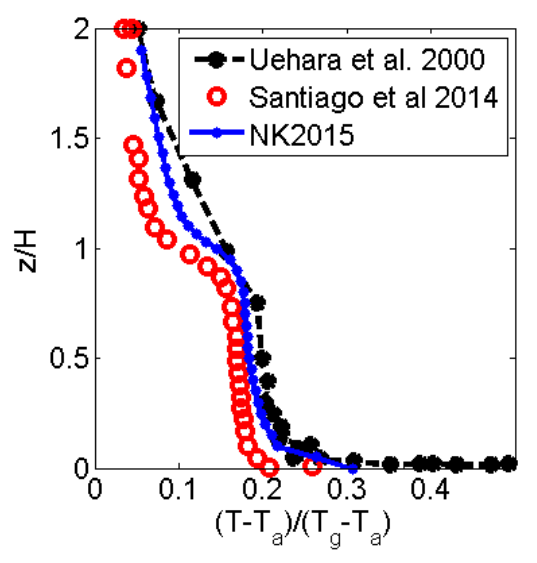

Figure 5: Vertical profile of normalized temperature $\left(T-T_{a}\right) /\left(T_{g}-T_{a}\right)$ at Location B (Fig. 1) for WMLES model.

For a more comprehensive validation of simulated temperature, the experimental data from Uehara et al. [11] have been used (similar to previous studies by Xie et al., [46]; Park et al., [21]; and Santiago et al. [63]). The experimental configuration is so that the temperature of the ground is higher than the ambient air temperature. The simulations are conducted for a bulk Richardson number $\left(R i_{b}=g H\left(T_{a}-T_{g}\right) / T_{a} U_{b}^{2}\right)$ of 0.21 , where $T_{g}$ is the canyon floor temperature. Here, this configuration is modelled with the same resolution and same geometrical simulation setup as in Section 3 to validate the model under the same conditions as in the simulations used to produce the results for this paper. The temperature profile calculated with the LES in this study (NK2015 in Figure 5) shows improved accuracy compared to the RANS simulation of Santiago et al. [63], with normalized mean square error (NMSE) of $0.97 \times 10^{-2}$ compared to NMSE value of $1.3 \times 10^{-2}$ in [63]. The temperature profile is well reproduced in the street canyon with MAPE of 9 percent, while the temperature is underestimated above the building height and close to the floor and can be attributed to the grid resolution in the boundary layer region as discussed before. 


\section{Results}

\subsection{Thermal forcing in the canyon}

Previous studies pointed to the importance of thermal forcing on microclimate, but limited their evaluation to uniform heating of merely one surface, which is unlikely in realistic conditions. The significance of three-dimensional non-uniform heating is analyzed in this section. For reference, a neutral case (called "N" onward) is simulated where the solar forcing is zero and the temperature of all surfaces is equal to the inlet air temperature at $288.4 \mathrm{~K}$.

The sunlit wall configuration and solar heat flux intensity depends on time, location, and street canyon geometry. Figure 6 shows time-averaged snapshots at different Time of Day (ToD) of non-uniform surface temperature on building walls. Results in the right column of the figure are averaged over the $H \times H \times H$ volume downwind of the center building in the spanwise canyon, hereonafter referred to as "Building Canyon" and indicates the non-uniform ground heating in the spanwise canyon. At 0600 and 0800 PST, the leeward wall of the building is partially heated, while the magnitude of solar heating and the ratio of the heated to overall wall surface at 0800 PST is significantly larger. From 0800 to 1000 PST, the transition from nonuniform to uniform leeward heating occurs, and the ground surface in the building canyon is also partially subjected to solar heating (shown in the right column). Solar heating is near uniform and mostly restricted to the ground and roof surfaces at noon (solar noon is at 1150 PST). Afterwards, wall heating transitions to windward and the magnitude of solar insolation and ratio of the heated wall decrease from 1400 to 1800 PST. The ground surface in the streamwise canyon (E-W outside the building canyon) and roof surfaces encounter only minor shadowing throughout the day, except for 0600 and 1800 PST when the solar altitude is low. Additionally, roof heating is significantly larger than uniform wall heating at 1000 and 1400 PST. Similar to east and west walls, north and south walls also go through heating transition, affecting the flow structure and temperature distribution in the building canyon.

\subsection{Characterizing momentum versus buoyancy forcing}

Traditionally, the bulk Richardson number calculated based on the vertical temperature gradient is used to quantify the thermal instability in the street canyon. Few studies (including [63]) used the total surface heat flux for calculating the buoyancy parameter. The characteristics of the thermally stratified flow also depend on the canyon aspect ratio. Fernando et al [64] assumed that $\frac{\partial u}{\partial x} \sim \frac{\partial w}{\partial z}$ and showed that the defined buoyancy parameter becomes proportional to the reciprocal of aspect ratio squared $(W / H)^{2}$ for a $2 \mathrm{D}$ canyon. Additionally, Dallman et al [65] introduced a non-dimensional buoyancy parameter, B, considering the canyon aspect ratio and temperature difference between walls; however this buoyancy parameter does not distinguish the directionality of the temperature gradient. Using CFD of a 2D street canyon, Magnusson et al [66] also concluded that the results are dependent on the canyon aspect ratio as B varies, despite the fact that AR is included in the buoyancy parameter, B.

As of yet, the methods to characterize thermal forcing neglect the horizontal temperature gradient, and fall short in comprehensive characterization of the flow. Here we define two non-dimensional numbers to convey more information about the directionality of thermal forcing in relationship to the canyon vortex in addition to the bulk vertical instability in the canyon. Canyon aspect ratio appears in the definition of horizontal Richardson number, however the effect of geometry is not analyzed in this paper. 

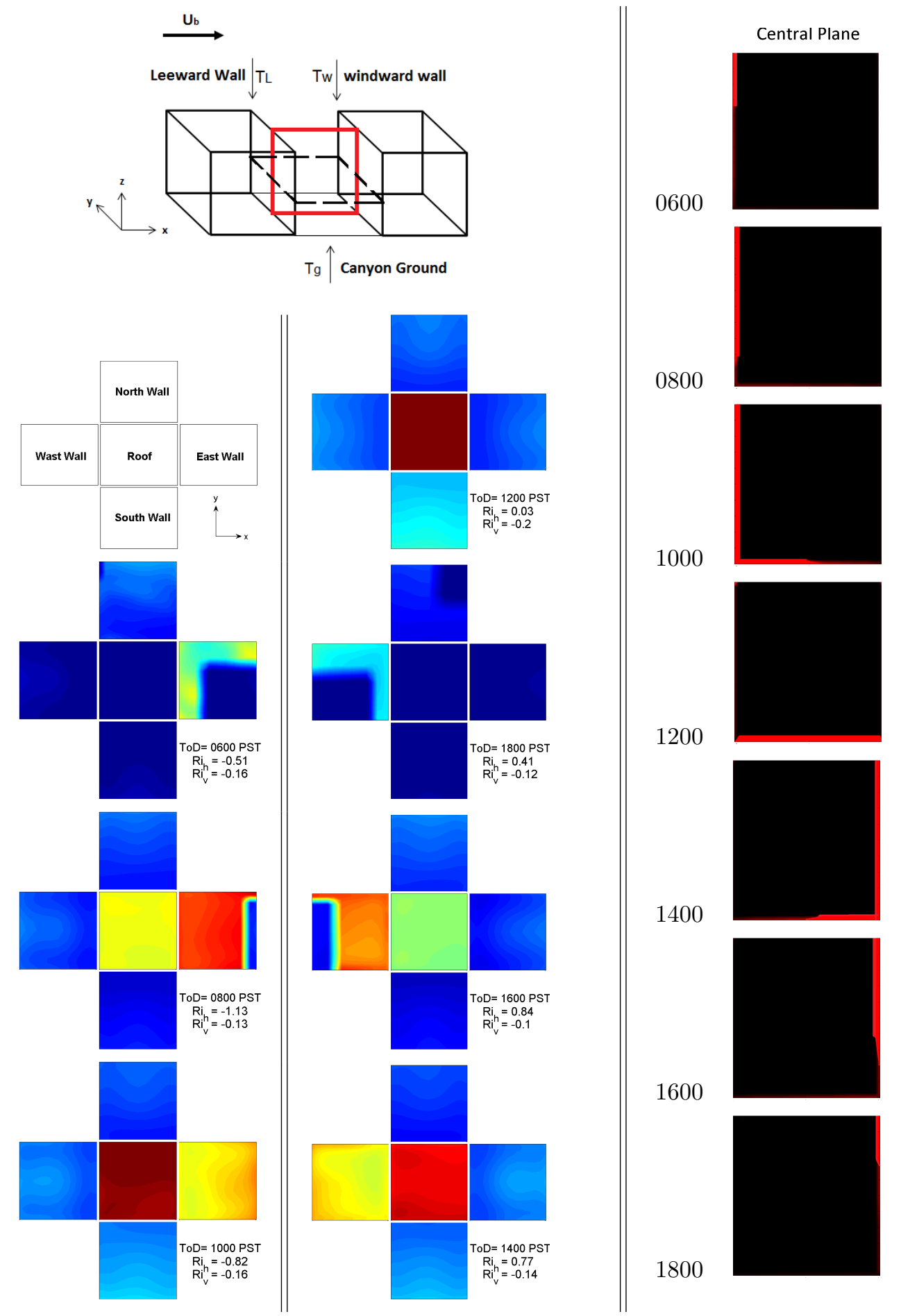

Figure 6: Schematic of surface temperature distribution at building walls at different Times of Day (ToD). The three dimensional geometry of buildings and the spanwise canyon between them is shown on the top left; with the red square indicating the vertical plane at the center of the building canyon. The right column displays sunlit surfaces in that plane including the ground surface which is not shown in the left column and can be used as a reference for following figures. The dashed square in the top left figure indicates the horizontal plane at $z / H=0.5$ used later. 
The bulk Richardson number, $\mathrm{Ri}_{\mathrm{v}}$, indicates the atmospheric stability in the vertical direction

$$
R i_{v}=\frac{g H}{\left(U_{b}^{2}\right)} \frac{\left(\overline{T_{H}}-\overline{T_{g}}\right)}{T_{a}}
$$

where $g=9.81 \mathrm{~ms}^{-1}$ is the gravitational acceleration, $T_{H}$ is the air temperature at roof level, $T_{g}$ is the temperature of the ground surface inside the building canyon (Fig. 6), $T_{a}$ is the inflow air temperature, and $U_{b}$ is the bulk wind velocity in the streamwise direction. $T_{g}$ and $T_{H}$ are averaged over the building canyon and in time (the two spanwise canyons downwind and upwind of the center building as described in Section $4.2)$.

However, since the orientation of the heated wall affects the flow field inside the canyon, bulk Richardson number is not sufficient to characterize the flow field. In the micro-climate of the urban canyon, the temperature gradients in horizontal directions $\left(\frac{\partial T}{\partial x}\right.$ and $\frac{\partial T}{\partial y}$ in the span-wise and stream-wise canyon, respectively), as well as the wind direction are expected to impact the strength of the canyon vortex. In the building canyon with wind direction as shown in Figure 1, the ratio of these terms forms a new buoyancy parameter as

$$
\begin{aligned}
R i_{h} & =\frac{\frac{g}{\bar{T}} \frac{\overline{\partial T}}{\partial x}}{\left(\overline{\frac{\partial U}{\partial z}}\right)^{2}} \sim \frac{\frac{g}{T_{a}} \frac{\left(\overline{T_{L}}-\overline{T_{W}}\right)}{W}}{\frac{U_{b}^{2}}{H^{2}}} \\
& =\frac{g H}{U_{b}^{2}} \frac{\left(\overline{T_{L}}-\overline{T_{W}}\right)}{T_{a}} \frac{H}{W},
\end{aligned}
$$

where $T_{W}$ and $T_{L}$ are the averaged surface temperature on windward and leeward walls (here west and east), respectively. $\mathrm{Ri}_{\mathrm{h}}$ therefore indicates the effect of differential solar heating and also incorporates the effect of canyon aspect ratio $H / W$. Additionally, if the wind direction is not aligned with the streamwise canyon, bulk velocity $\left(U_{b}\right)$ should be multiplied by $\cos \alpha$, where $\alpha$ is the deviation angle (in the clockwise direction) from the stream-wise canyon. $R i_{h}$ combined with the commonly used bulk Richardson number $R i_{v}$ introduces a new potential way of comprehensively characterizing the flow under realistic surface heating.

Figure 7 shows the variation of $\mathrm{Ri}_{\mathrm{v}}$ and $R \mathrm{i}_{\mathrm{h}}$. $\mathrm{Ri}_{\mathrm{v}}$ ranges from -0.1 to -0.21 under unstable conditions between 0600 to 1800 PST, which is in the range of values reported by Nakamura and Oke [7] in urban field observations. The magnitude of $R i_{v}$ peaks at 1200 PST when ground heating peaks. The diurnal cycle of $R i_{v}$ deviates from what would be expected in reality for the following reason. The inlet air temperature, $T_{a}$, is set to the hourly air temperature from TMY3 data, and $T_{g}$ follows the same diurnal pattern as $T_{a}$ (not shown). This is partially an artifact of our simulation setup; steady-state simulations at different ToD ignore prior heat storage in the ground that cause $T_{g}$ to exhibit a greater amplitude than $T_{a}$ in reality. However, our simulation setup allows us to focus on the effect on thermal forcing due to solar insolation only, and not to combine the additional effect of ground thermal inertia especially in afternoon hours. At 0600 and $1800 \mathrm{PST}$, in the absence of roof heating $T_{g}$ is relatively warmer than $T_{H}$ compared to 0800 and 1600 PST, resulting in larger atmospheric instability. There is some asymmetry in the graph due to the solar noon occurring before 1200 PST.

The $R i_{h}$ exhibits large variations with ToD. The sign of the $\mathrm{Ri}_{\mathrm{h}}$ indicates whether the canyon is subject to windward or leeward heating as a result of solar azimuth changes. The sign of $\mathrm{Ri}_{\mathrm{h}}$ changes after 1200 PST while its magnitude is symmetrical about noon. $\mathrm{Ri}_{\mathrm{h}}$ peaks when the temperature gradient between building walls reaches its maximum. $R i_{v}$ and $R i_{h}$ are both zero for the neutral case $(\mathrm{N})$. 


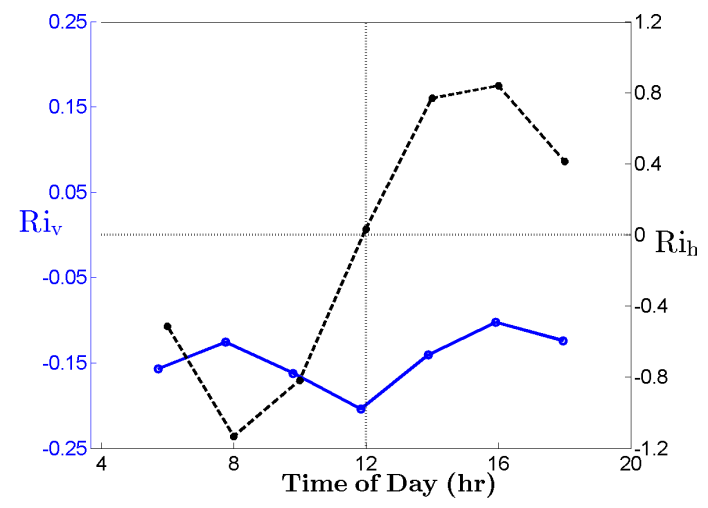

Figure 7: Richardson number variation with Time of Day (ToD). Blue solid line represents the vertical Richardson number $\left(R i_{v}\right)$ corresponding to the left vertical axis, and black dashed line shows the horizontal Richardson number $\left(R i_{h}\right)$ corresponding to the right axis.

\subsection{Flow and thermal field in the building canyon}

The flow and heat transport as influenced by the three-dimensional non-uniform heating is analyzed. This section is structured as follows. First, the effect of 3D thermal forcing on air temperature inside the canyon is analyzed (see Fig. 8 and 9 later). Then, the flow field and the canyon vortex are compared at different ToD, through vector and contour plots of time-averaged velocity components as well as pressure fields in the spanwise (N-S) and streamwise (E-W) cross sections (Figs. 10 - 13). Lastly, time-averaged turbulent kinetic energy (TKE) is calculated in order to measure the intensity of turbulence as effected by combined forcing of buoyancy and shear (Fig. 14). The results are calculated at the vertical and horizontal plane in the center of the spanwise canyon (red and dashed squares, respectively, in Fig. 6) and time-averaged over $1500 \mathrm{~s}$ corresponding to at least 15 times the integral time scale (Section 2.4). The results are also compared with previous studies with uniform heating of one or two urban surfaces in 2-D and 3-D canyons.

The effect of wall heating on air temperature inside the building canyon is shown in Figure 8 and 9 . The spatial distribution of air temperature in the vertical plane (Fig. 8) demonstrate the transition from leeward (E-Facing) to windward (W-Facing) heating in addition to effects originating from the roof, while the results in horizontal cross sections (Fig. 9) allow the study of thermal forcing from the streamwise street canyon. At 1200 PST, the heat advected from the roof surface causes a larger temperature increase in the canyon than the convection from the ground (Fig. 8). Additionally, at 1200 PST the south-facing wall is warmest due to the southerly sun position and heat is advected into the spanwise canyon from the south (Fig. 9 1200 PST). Therefore air temperature and flow field (described in more detail below) at 1200 PST deviates from the widely studied case of canyon ground heating (for example [17] and [22]).

The distinction between leeward versus windward wall heating is also apparent on the air temperature inside the building canyon. When the leeward wall is heated at 0800 and 1000 PST, elevated temperatures are more concentrated near the leeward wall and the heat is primarily transported vertically. For example at 0800 and 1000 PST the leeward heating adds to the effect of roof heating in heating the air at the top of the canyon, while the effect of partial ground heating at 1000 PST is less significant. In the windward heating cases, however, higher air temperature is observed within the canyon suggesting enhanced heat transfer and mixing inside the canyon (Fig. 9). This behavior is also reported in the study by Cai et al. [67] that analyzed the combined effect of uniform roof and wall heating in a 2-D canyon.

The contour plots of mean velocity magnitude superimposed by velocity vectors are shown in Figures 10 and 11, followed by the plots of static pressure (Figs. 12 and 13). Throughout the day, only one vortex is observed in the vertical plane and the vortex center is located above $z / H=0.75$. While the windward wall is strongly heated in the afternoon $\left(R i_{h}=0.79\right.$ and 0.83 at 1400 and 1600 PST, respectively) the thermal forcing from the heated wall is not enough to create a secondary vortex in the 3-D canyon. Our finding of 


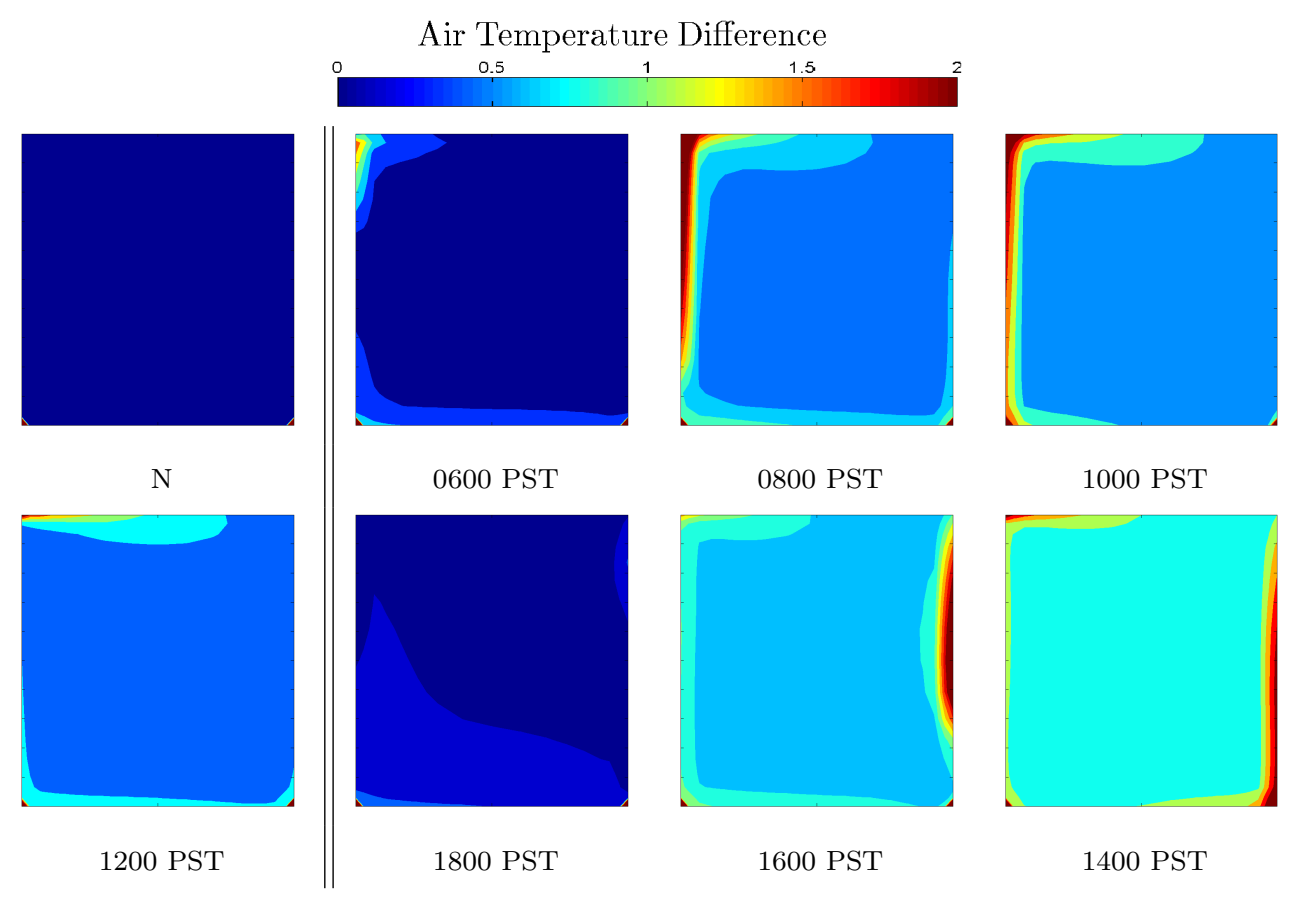

Figure 8: Contour plots of the air temperature anomaly from the inlet air temperature $\left(\left(T\left(x^{-}, z, t\right)-T_{a}(t)\right)\right)$ at different ToD. Results are calculated at the vertical plane in the building canyons (red square in Fig. 6) and timeaveraged over $1500 \mathrm{~s}$. The plots are arranged such that axisymmetrically opposed horizontal thermal forcing, i.e. similar $\left|R i_{h}\right|$ as at 1000 and 1400 PST, are in one column. The left column shows the neutral case (N) and noon where wall heating is symmetric.

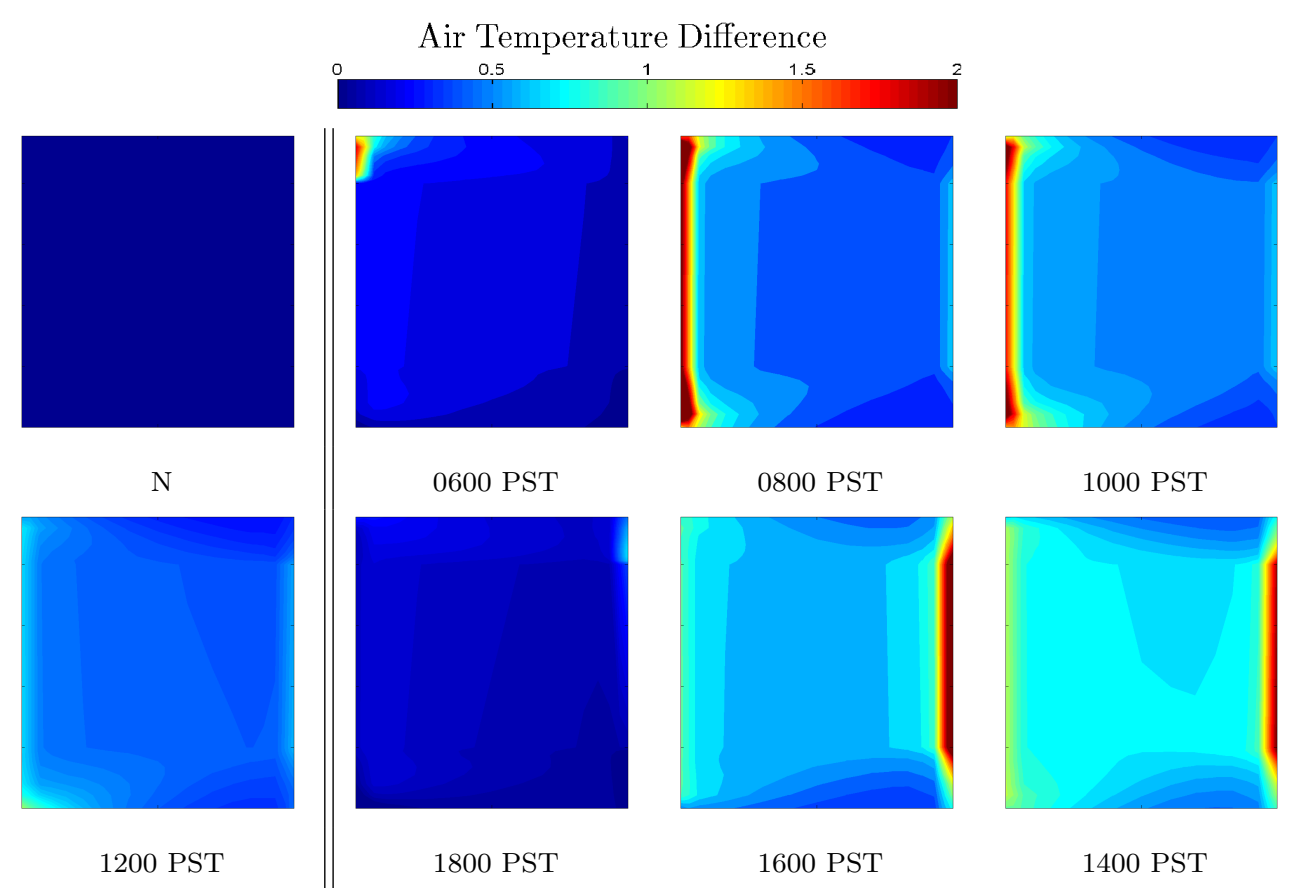

Figure 9: Similar to Figure 8 for the horizontal cross section at $z / H=0.5$ (dashed square in Fig. 6). 
a single vortex is consistent with field measurement of Offerle et al [68] who did not observe a secondary vortex near the windward wall as reported in previous numerical studies. The wind tunnel experiment of Allegrini et al [22] and numerical simulation by Kim and Baik [17] that analyzed uniform ground heating suggest that the vortex intensity increases with heating in canyons with small aspect ratio (range of 1 to 1.2 ). In our simulations, building aspect ratio is in that range $(\mathrm{AR}=1)$ and the temperature gradient between ground and air at roof level at $1200 \mathrm{PST}$ is approximately $6 \mathrm{~K}\left(R i_{v}=-0.2\right.$ and $\left.R i_{h}=0\right)$. However, the vortex intensity decreases at 1200 PST in contradiction with the uniform ground heating scenario of [17] and [22]. We hypothesize that the presence of roof heating causes the vortex intensity to decrease and the center location is shifted closer to the windward wall.

In comparison with the uniform ground/roof heating at $1200 \mathrm{PST}$ and the neutral case (N), two regimes are observed:

a) In near neutral conditions of 0600 and 1800 PST without significant roof or ground heating, the flow structure is consistent with the neutral simulation $(\mathrm{N})$ as expected. The flow field in this regime is only affected by the strength and orientation of the wall thermal forcing. While the vortex strength slightly increases for leeward heating (0600 PST), the fact that the changes in the flow are minor at 0600 versus 1800 PST despite the large variation in $R i_{h}$ suggest a dominance of inertial over buoyancy forcing. The weak effect may also be a result of the location of the heated wall. While the $R i_{h}$ consider the average wall temperature, natural convection scales with $L^{3}$ and therefore the short section of heating (Fig. 6).

b) In unstable conditions between 0800 to 1600 PST the flow is consistent with the 1200 PST simulation. The flow field in this regime is characterized by the superposition of wall, ground and roof heating in both building canyon and the streamwise canyon. Although the distinction between windward and leeward heating is apparent in the temperature fields (Figure 8 and 9), apparently other factors dominate the flow field as the vortex in Fig. 10 appears unchanged. For example, throughout the day the ground in the streamwise (E-W) canyon is mostly unshaded. However large velocity in the streamwise canyon results in larger convection therefore lower temperatures at the floor of the streamwise canyon and this effect is commonly neglected in the literature. The effect of this temperature gradient in the spanwise direction is then seen in the horizontal pressure gradient (Figure 13). Additionally, the temperature difference between the air above the heated roof, and air at roof level averaged inside the canyon is dynamic during the day. For windward wall heating $\left(R i_{h}>0\right)$, larger horizontal (streamwise) temperature gradient exists at the roof level, therefore a lower pressure gradient is observed inside the canyon (upper part of the building canyon except for the top right corner) compared to the leeward heating cases. This horizontal pressure gradient results in higher velocity at the roof level and higher kinetic energy entering the building canyon in windward heating cases $\left(R i_{h}>0\right)$. Therefore, while considering the wall forcing only a weaker vortex would be expected, holistic consideration of the three-dimensional thermal forcing shows that other effects dominate. However, the location of the canyon vortex is affected by the wall heating. For leeward heating $\left(R i_{h}<0\right)$ the center of the canyon is closer to the windward wall. We hypothesize that the cooler air towards the top of the canyon (Fig. 10, 0800 and 1000 PST versus 1600 and 1400 PST) is partially caused by buoyancy from the leeward wall causing a weakening of the vertical pressure gradients near the canyon top (Fig. 12) and therefore less flow into the canyon from the top.

Additionally, when comparing the mean vertical velocity for cases with symmetrically opposed wall heating (not shown), the downdraft velocity is smaller compared to the neutral case at 1400 and 1600 PST due to windward heating that counteracts momentum forcing. However, the downward vertical velocity is larger at 1400 and 1600 PST than during hours with leeward heating. This can again be explained by the increase in vortex intensity due to the horizontal pressure gradient effect for the windward heating cases that brings more momentum into the canyon. The region of updraft velocity is also larger for negative $R i_{h}$ (leeward heating).

The three-dimensionality of thermal forcing is also apparent in horizontal cross section of the building canyon $(z / H=0.5$ in Fig. 11). Two counter-rotating vortices are observed. While symmetrical in the neutral (N) case, the heat advected from the warmer wall in the streamwise canyon (North or South, see also section 5 and Fig. 6) affects the size and strength of the vortices in the horizontal plane. For example, the northfacing wall is warmer at 0600 and 1800 PST and convection of heat transported from the north wall creates a low pressure region in the streamwise canyon that weakens the pressure differential to the spanwise canyon 


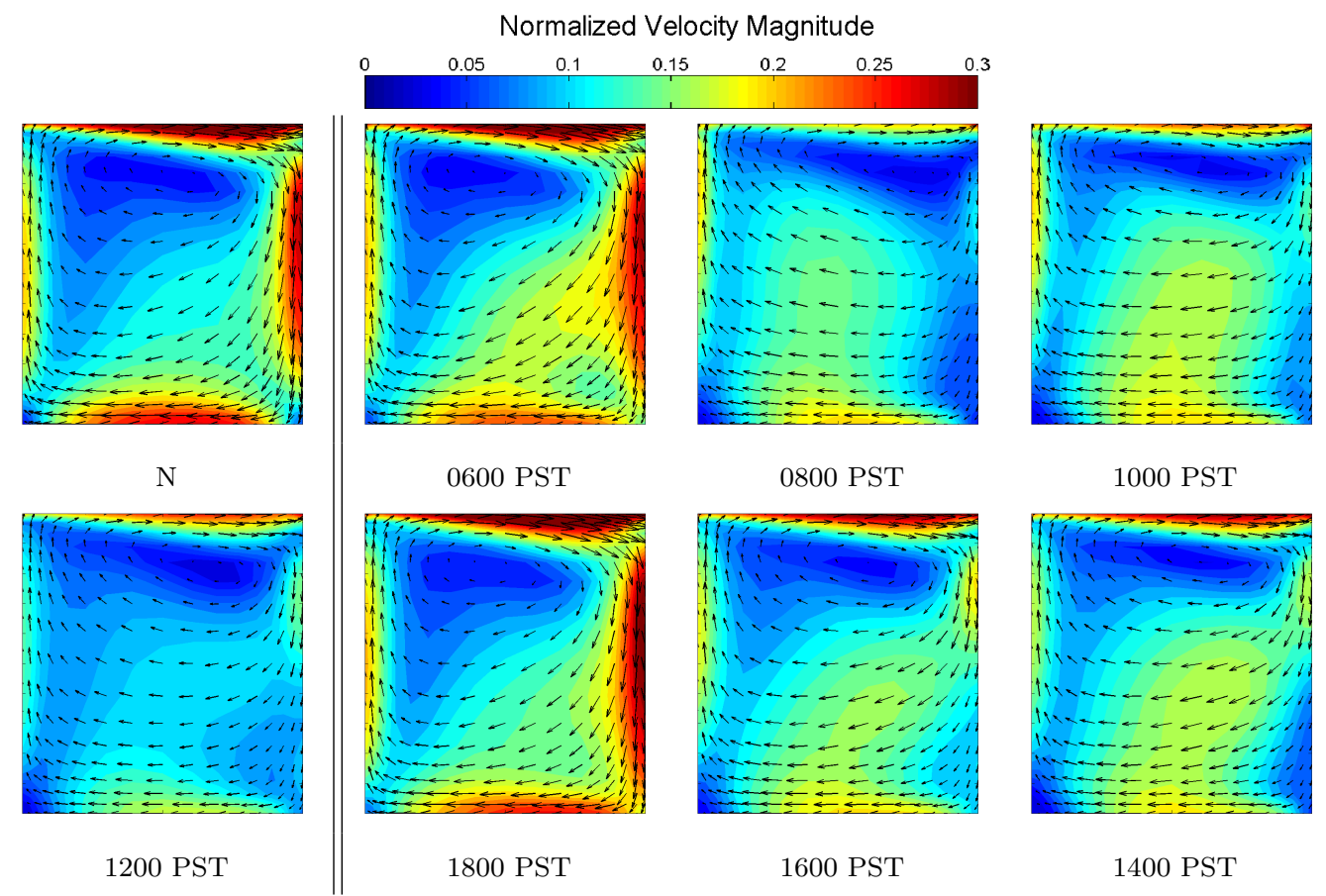

Figure 10: Contour of mean velocity magnitude normalized by bulk wind velocity, $\frac{\bar{U}}{U_{b}}$, overlaid by the mean velocity vector field at different ToD. Results are calculated at the vertical plane in the building canyon and time-averaged over $1500 \mathrm{~s}$.

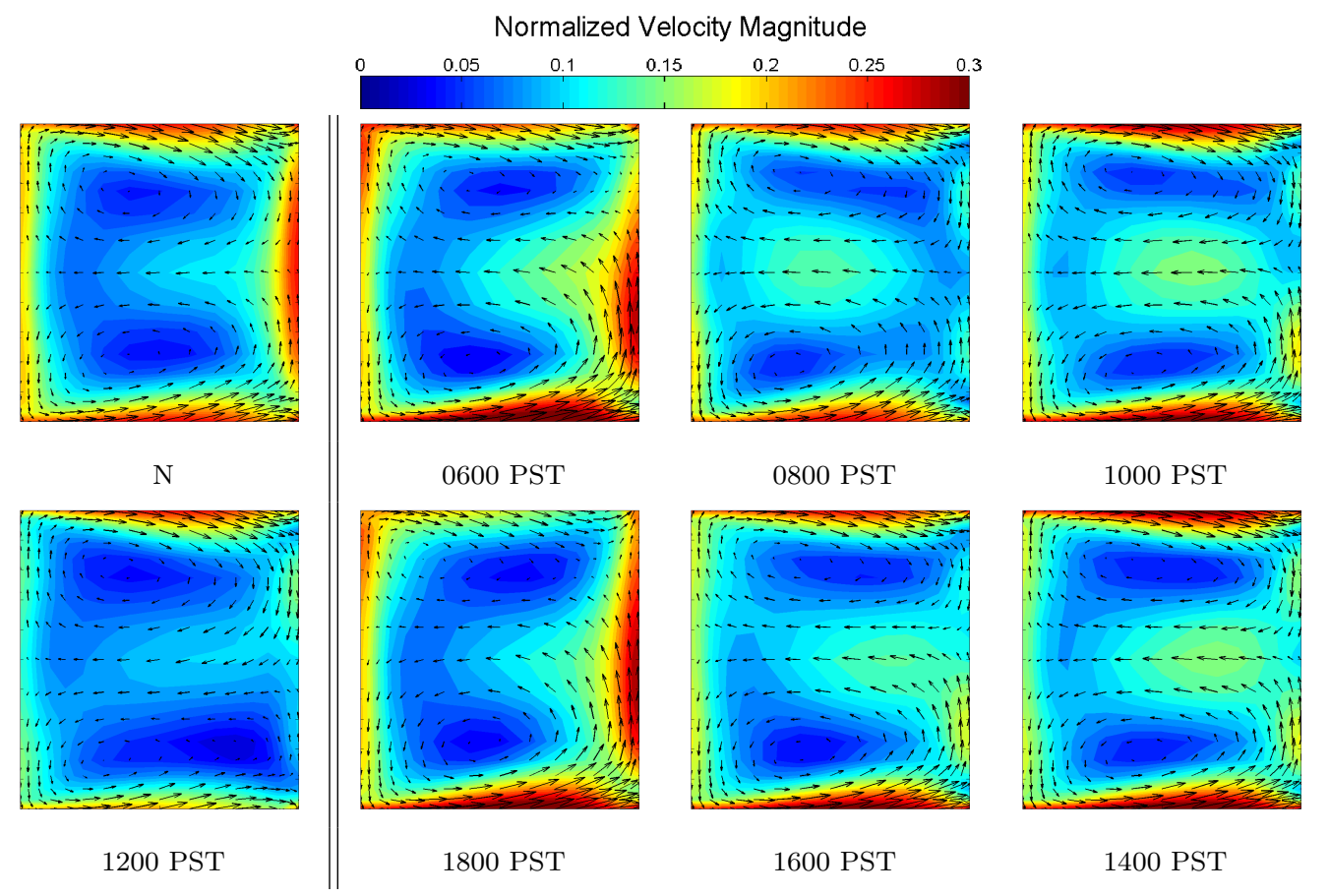

Figure 11: Similar to Figure 10 at the horizontal cross section shown in figure 6. 
resulting in a weaker north vortex. Similar albeit weaker behavior is observed at 0800 and 1600 PST (weak north wall heating, Fig. 6) as well as 1200 PST, when south wall heating affects the southern vortex. At 1000 and 1400 PST, however, strong east-west wall heating in addition to ground heating seems to overcome the effect of the warm south wall and the two vortices are more symmetrical. The asymmetric behavior of flow in the horizontal plane is further analyzed in Section 4.5 in the context of air exchange from the building canyon.

Figure 14 shows the contour plots of turbulent kinetic energy (TKE) at the central vertical plane of the cross-stream canyon. In all cases, the highest TKE is observed in the area of large shear near the windward wall where the air enters the building canyon from above. Even when shear $\left(\frac{d u}{d x}\right)$ is smaller near the windward wall (0800 to 1600 in Fig. 10), TKE remains elevated there especially for the cases with windward heating (1400 and 1600 PST), presumably due to intermittent (counter-flow) buoyant updrafts. In leeward and ground heating cases, buoyancy acts in the same direction as the flow, adding to the kinetic energy in the building canyon while the windward heating causes larger TKE near the heated wall due to opposing thermal and momentum forcing. In the second regime (from 0800-1600 PST), the TKE inside the canyon increases for windward, leeward and ground heating (in that order). Horizontal cross-sections of TKE (not shown) support these conclusions. 


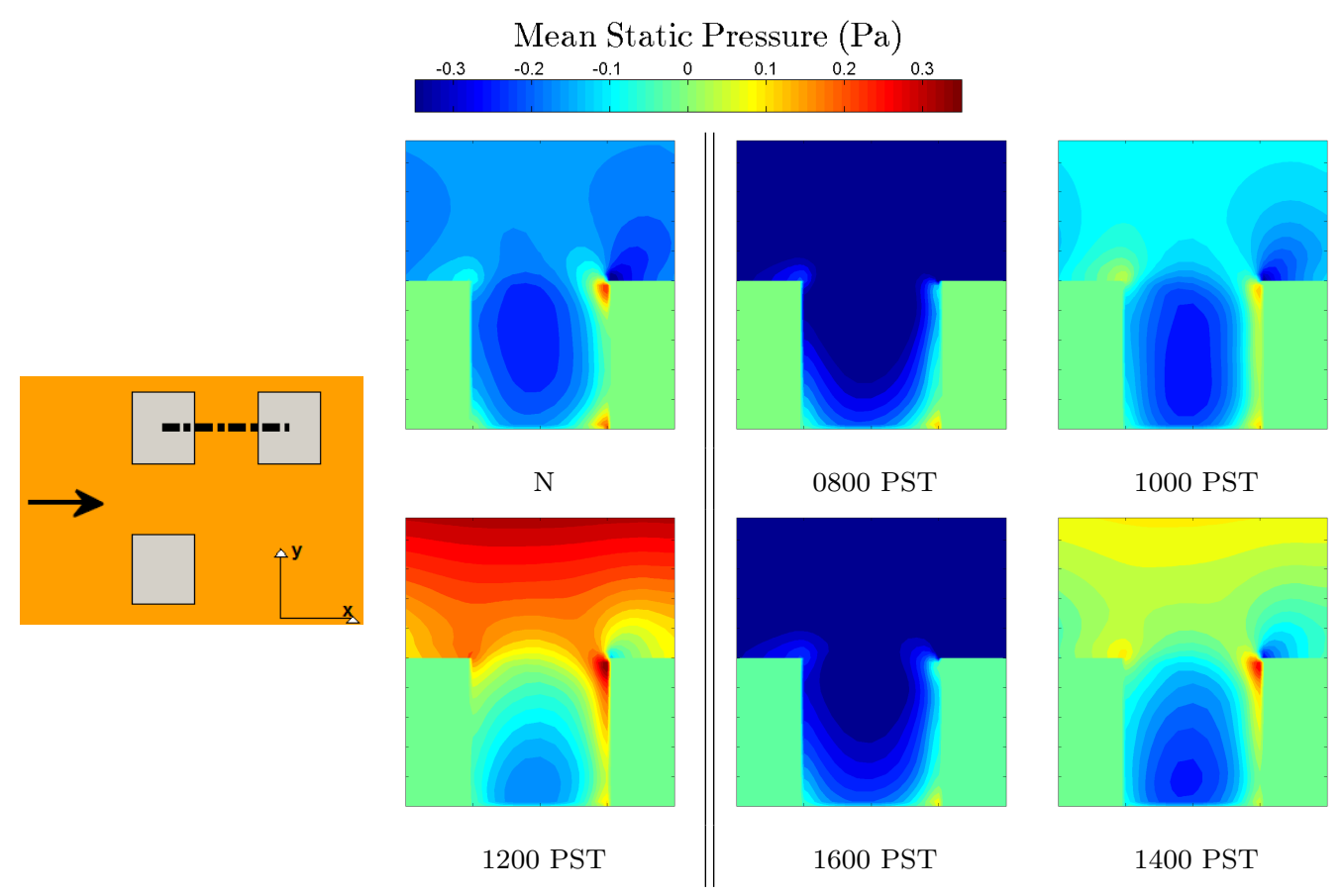

Figure 12: Contour plots of mean static pressure $(\mathrm{Pa})$. Results are calculated at the vertical plane $(\mathrm{x}-\mathrm{z})$ in the building canyons and time-averaged over $1500 \mathrm{~s}$.

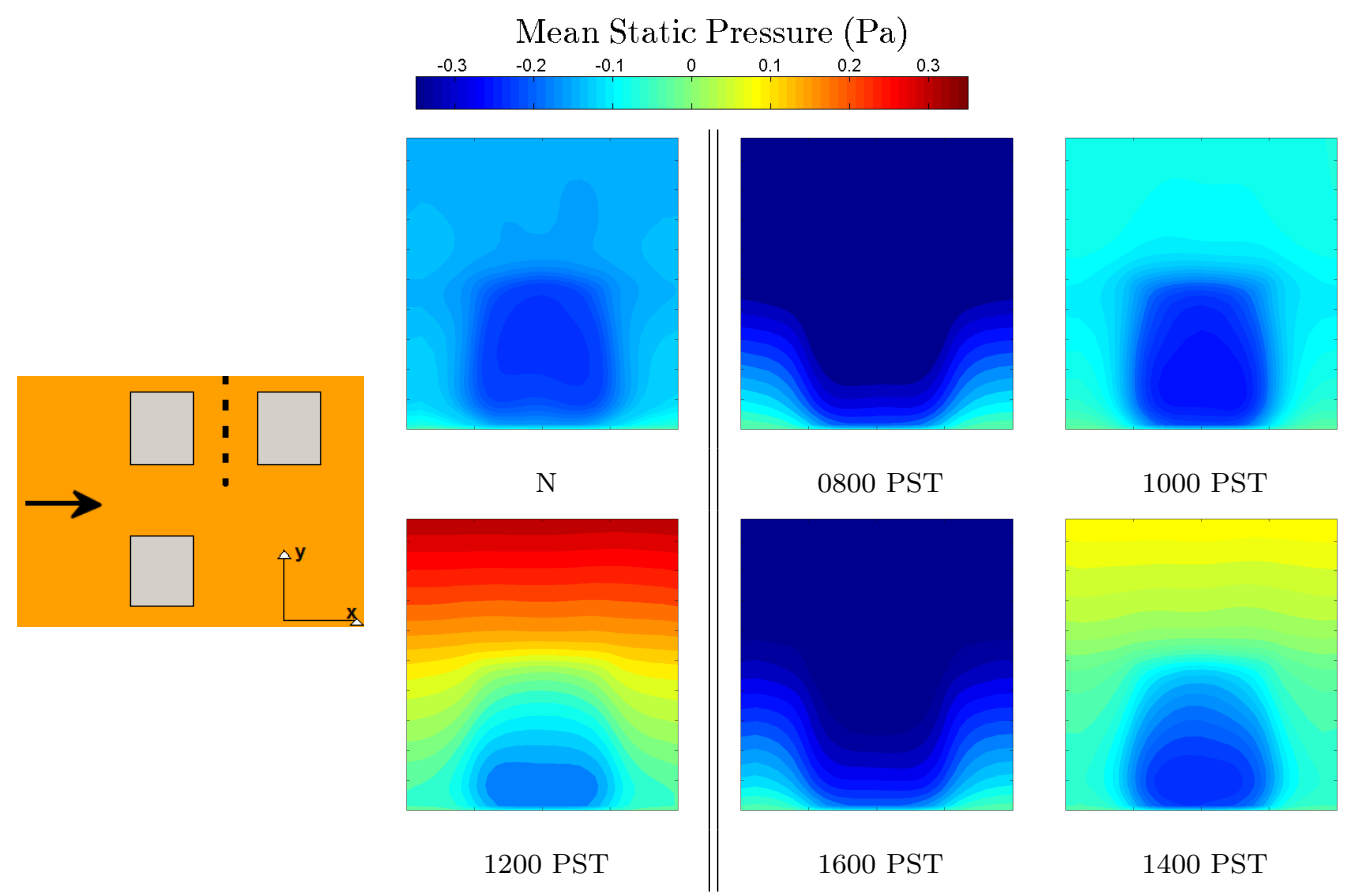

Figure 13: Contour plots of mean static pressure $(\mathrm{Pa})$. Results are calculated at the vertical plane $(\mathrm{y}-\mathrm{z})$ in the building canyons and time-averaged over $1500 \mathrm{~s}$. 


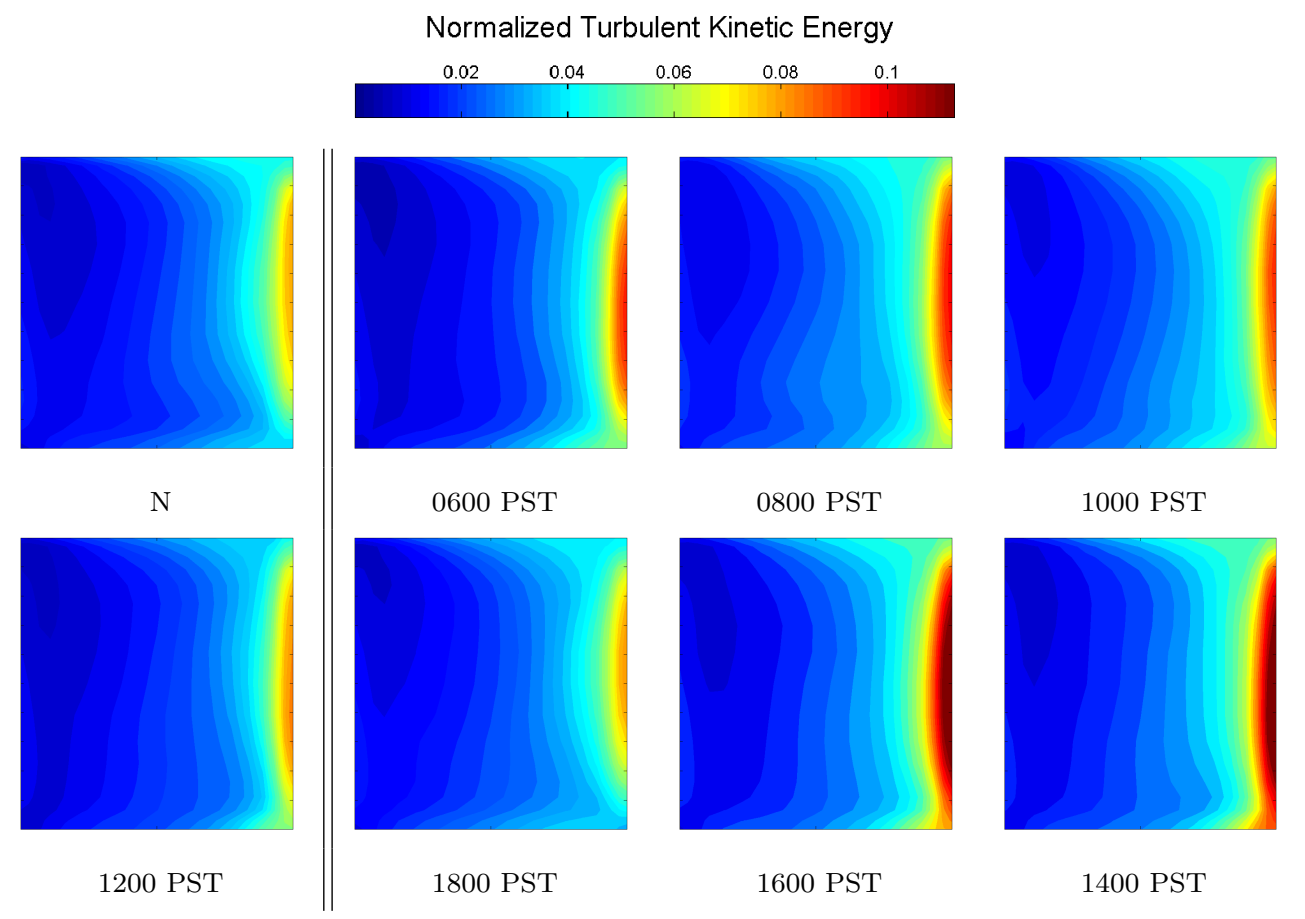

Figure 14: Contour plots of Turbulent Kinetic Energy normalized by $U_{b}^{2}$ with Time of Day (ToD). Results are calculated at the vertical plane in the span-wise building canyons (Fig. 6) and time-averaged over $1500 \mathrm{~s}$. The plots are arranged such that axisymmetrically opposed horizontal thermal forcing, i.e. similar $\left|R i_{h}\right|$ as at 1000 and 1400 PST, are in one column. The left column shows the neutral case $(\mathrm{N})$ and noon where wall heating is symmetric.

\subsection{Convective heat transfer coefficient}

The accurate evaluation of CHTCs on exterior building surfaces requires that the grid is refined in the boundary-layer region in order to obtain $\mathrm{y}+$ (dimensionless wall distance) value of about 1 [36, 37]. However, due to the computational cost associated with the low resolution of the LES, this requirement cannot be met in the current study, therefore it is expected that the limited wall resolution may impact the CHTC accuracy, especially in the areas near the edge of building walls (as shown by Figure 4 in the Section 3). However, the relative variation of CHTC at different times of day is presented to evaluate the sensitivity of CHTC to the non-uniform surface heating. This evaluation can further improve our understanding on the relative importance of thermal and momentum forcing inside the canyon on the outdoor ventilation performance.

In this section, we analyze local Nusselt number distributions on the center building (Fig. 1) under realistic wall heating. The non-dimensional parameter $\sigma$ is defined for measuring distance along building walls in the peripheral directions, which is the distance from the South-West corner of the building moving around the building walls in the clockwise direction (Fig. 15). CHTC is then averaged in the vertical direction.

In general CHTC is larger in the west (windward) wall compared to east (leeward) wall, but the difference depends on the ToD. For example, when the windward wall is heated (1400 - 1800 PST), the associated buoyancy near the heated wall, in addition to the enhanced vortex strength due to the lower pressure gradient inside the canyon, results in the largest CHTC at the windward wall, while reducing the CHTC at the leeward wall. North and south walls have exhibit higher CHTC since the velocity is higher in the stream-wise canyon. A diurnal cycle is evident where ToDs with similar solar zenith angles and thus similar south wall insolation (e.g. 0800 and 1600) exhibit similar normalized CHTC on the South wall.

Convective Heat Transfer Coefficient (CHTC) at the roof surface (not shown) is also influenced by the horizontal and vertical Richardson number ( $R i_{h}$ and $R i_{v}$, respectively). The CHTC at roof for neutral, 0600, 

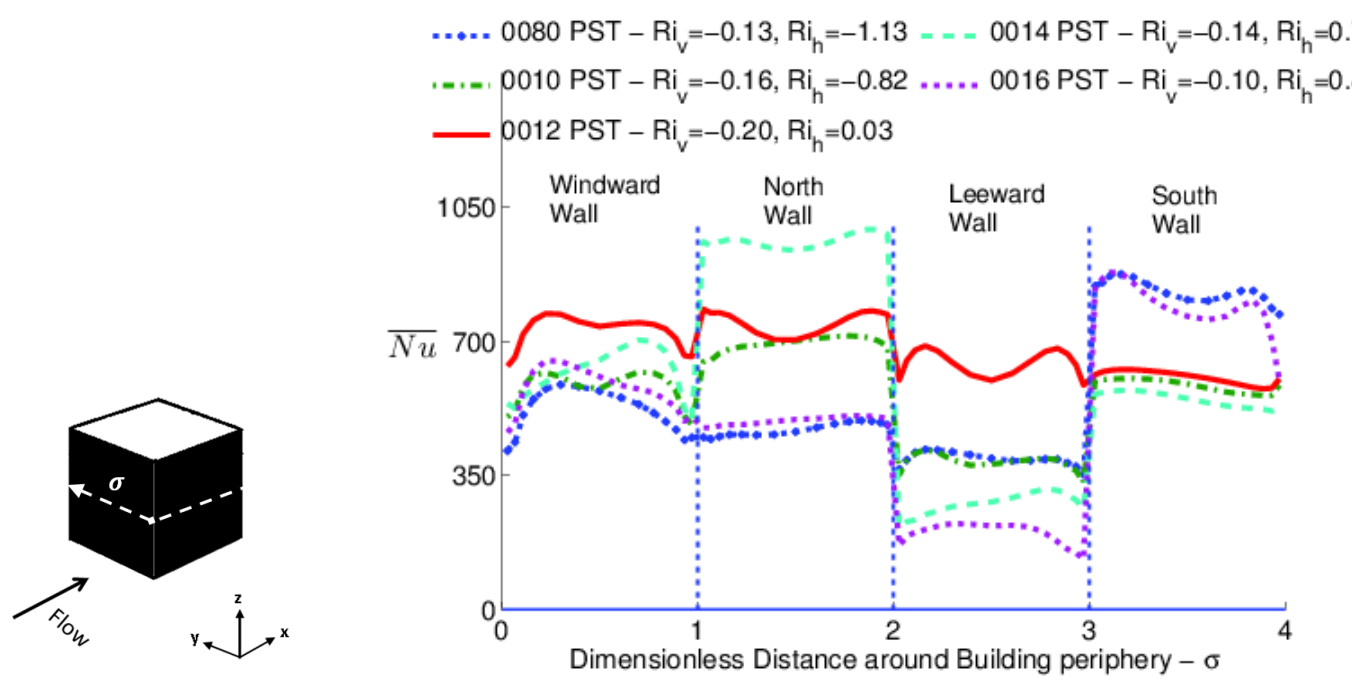

Figure 15: Local Nusselt number in the peripheral direction. For aesthetic reasons only selected ToDs are shown.

and 1800 PST simulations is smaller than at 1200 PST. Similarly, Nusselt number at roof level is higher for the windward heating cases (positive $R i_{h}$ at 1400 and 1600 PST) compared to leeward heating (negative $R i_{h}$ at 0800 and 1000 PST). For all cases, there is a local maximum in CHTCs at the upwind edge of the roof surface, where there is a localized high velocity.

\subsection{Street canyon ventilation and determinants of dispersion}

In this section, we examine the effect of non-uniform heating on air ventilation of the urban canyon. The concept of Air Exchange Rate (ACH) was introduced by Liu et al [44] and calculated as the area-weighted average of vertical velocity fluctuations at roof-level. The removal or entry of air is indicated by positive $(\mathrm{ACH}+)$ or negative $(\mathrm{ACH}-)$ air exchange rate, based on the direction of the fluctuating vertical velocity. In $2 \mathrm{D}$ street canyons, roof-level $\mathrm{ACH}+$ and $\mathrm{ACH}$ - balance due to the zero net vertical flux across the top of the street canyon. For 3D street canyons, Sun et al [69] observed a balance of positive and negative $\mathrm{ACH}$ in each ventilating face in the absence of buoyant forcing. However, when considering the asymmetrical thermal forcing of street canyons during daytime, air exchange through the different ventilating faces of the building canyon is likely unbalanced and unsteady (please refer to Figure 11 and the explanation on flow field in the horizontal cross section in section 4.3). Applying kinetic (mass) balance in the 3D street canyon, $3 \mathrm{D}-\mathrm{ACH}$ is defined by sum of air exchange rates along the top and sides of the canyon as

$$
\overline{<\mathrm{ACH}>}=\overline{<\mathrm{ACH}_{\mathrm{top}}>}+\overline{\left\langle\mathrm{ACH}_{\text {sides }}>\right.},
$$

where the overbar represents temporal averaging and the brackets spatial averaging as

$$
\begin{aligned}
<\mathrm{ACH}_{\text {top }}> & =\frac{\iint w_{+} \mathrm{d} x \mathrm{~d} y}{A_{\text {top }}}, \\
<\mathrm{ACH}_{\text {side } \pm}> & =\frac{\iint v_{ \pm} \mathrm{d} x \mathrm{~d} z}{A_{\text {side }}} .
\end{aligned}
$$


Here only velocities exiting the canyon are considered, i.e. $w_{+}$are positive vertical velocities along the top plane and according to the orientation of the side plane, the sign of spanwise velocity $(v)$ differs to represent air removal from the canyon. $A_{\text {top }}=A_{\text {side }}=H^{2}$ are the areas of top and side of the urban canyon (Fig. 16). To analyze the relative effects of mean flow and fluctuations on air removal from the building canyon, $\mathrm{ACH}$ is calculated both using the velocity and local velocity fluctuations (e.g. $v^{\prime}=v-\bar{v}$ ) and referred to as $\mathrm{ACH}_{\mathrm{w}}$ and $\mathrm{ACH}_{\mathrm{w}^{\prime}}$, respectively.

As additional measures of dispersion, turbulent kinetic energy (TKE) and kinetic energy are calculated and averaged over the cross-stream canyon.

$$
\begin{aligned}
<\mathrm{TKE}> & =\frac{\iiint\left(u^{\prime 2}+v^{\prime 2}+w^{\prime 2}\right) \mathrm{d} x \mathrm{~d} y \mathrm{~d} z}{\mathrm{~V}_{\mathrm{BC}}} \\
<\mathrm{KE}> & =\frac{\iiint\left(u^{2}+v^{2}+w^{2}\right) \mathrm{d} x \mathrm{~d} y \mathrm{~d} z}{\mathrm{~V}_{\mathrm{BC}}}
\end{aligned}
$$

$V_{B C}$ in the above equations is the volume of the space between two buildings.
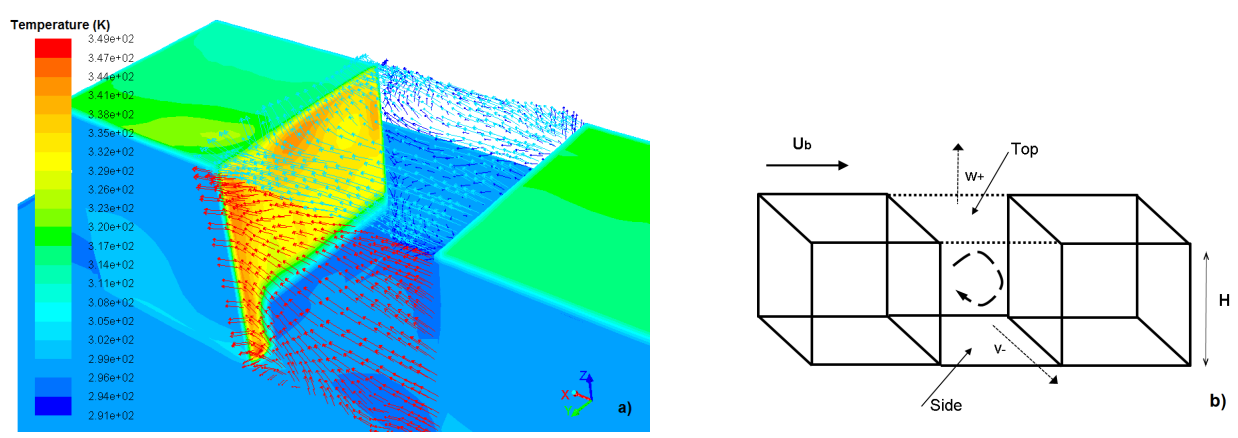

Figure 16: a) Contour of instantaneous temperature on urban surfaces (colorbar), overlaid by velocity vectors at the top and the side planes of the cross-stream canyon building space. Velocity vectors are colored based on their ventilating face. b) A sketch of the variables used to calculate the air exchange rates between the cross-stream canyon and the surrounding atmosphere.

Figure 17 shows the variation of air exchange rate and kinetic energy with time, normalized with $U_{b}$. In comparison with the neutral case $(\mathrm{N})$, thermal forcing generally increases the ACH, but by less than $10 \%$ and $\mathrm{ACH}$ is actually smaller at $1200 \mathrm{PST}$. The air exchange rate induced by velocity fluctuations $\left(A C H_{w^{\prime}}\right)$ is only $10 \%$ smaller than the total air exchange rate $\left(A C H_{w}\right)$, indicating the dominant effect of turbulence on ventilation from the street canyon. Air removal from the building canyon is enhanced for leeward heating compared to windward heating (positive $R i_{h}$ ). From 0600 to 1000 PST, when the strength of leeward heating increases and transition to ground heating occurs, ACH and TKE increase. Considering different ventilation faces during these hours (Fig. 18), most of the air is ventilated through the the top plane (at roof-level), while the north plane (side plane at positive y) is the lowest due to heating of air in the streamwise canyon adjacent to the north wall as described in Section 4.3. There is a significant ACH decrease at 1200 PST when the leeward heating effect is removed, and the roof heating is enhanced. The decrease in $A C H_{w}$ at the top plane shows the effect of roof heating on building canyon air removal (Fig. 18). Similarly, air removal from the south ventilation plane is decreased since south facing wall is warmer at 1200 PST (Fig. 8). Thermally induced and mechanically induced forcing are in opposite directions in the windward heating cases which explains the lower value of ACH at 1400 PST compared to 1000 PST. As the strength of windward heating and the area ratio of the wall exposed to direct sunlight decreases from 1400 to 1800 PST, ACH increases. 

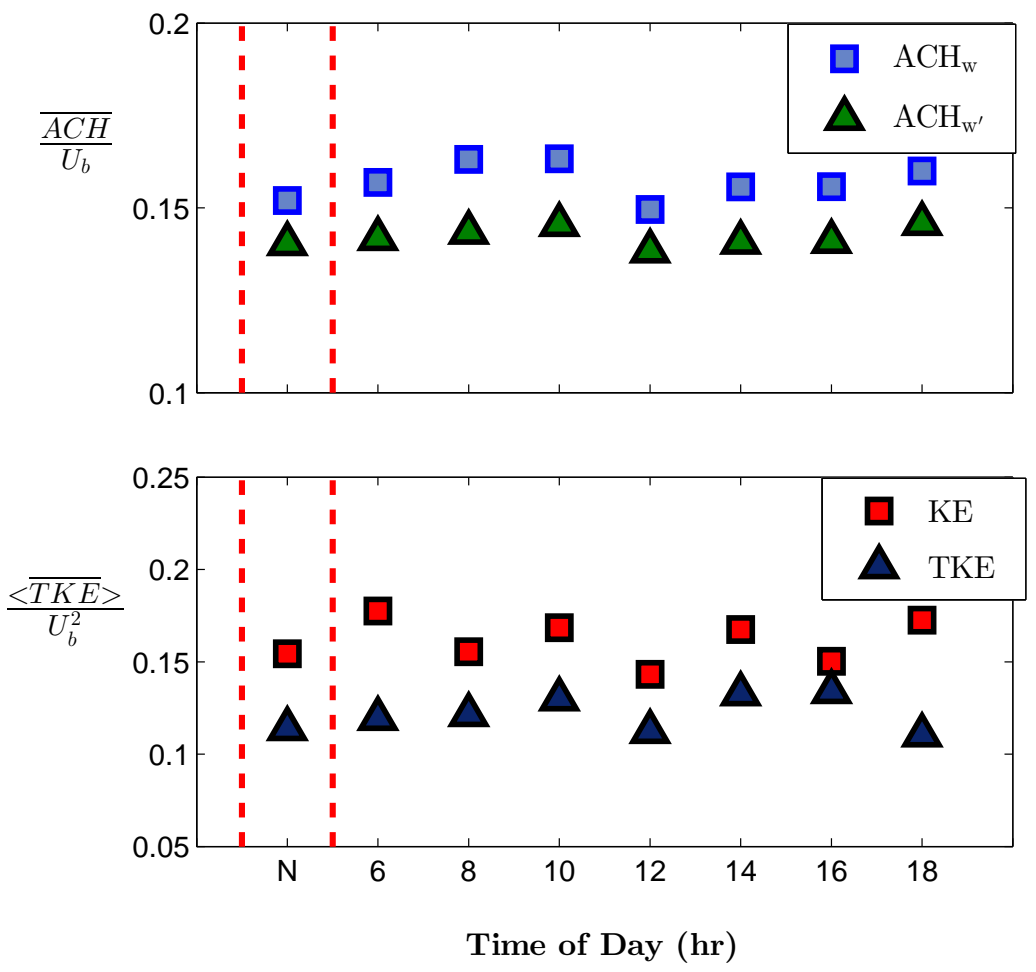

Figure 17: Total Normalized Air Exchange Rate at all ventilating surfaces (top) and Turbulent Kinetic Energy (bottom) in the building canyon as a function of ToD. The neutral case $(\mathrm{N})$ is shown as a reference and separated with red dashed lines.

Figure 17 (bottom) shows turbulent kinetic energy $(<\mathrm{TKE}>)$ and kinetic energy $(<\mathrm{KE}>)$ integrated over the volume between buildings. The magnitude of TKE for the windward heating cases is slightly higher as buoyancy opposes momentum forcing at the windward wall (Fig. 14) creating a intermittent flow patterns. The increase in TKE from the neutral case to 1000 PST can be explained by the increase in the surface heating and buoyancy-induced turbulence. The effect of TKE induced by roof heating is expected to have significant effects on building canyon TKE at near-solar-noon hours when roof is uniformly heated. TKE is not correlated with the orientation of the heated wall, rather it depends on the strength of overall wall heating in the building canyon. Kinetic energy does not follow the same pattern as ACH.

\section{Conclusions}

A three dimensional configuration of a compact urban environment is simulated with realistic surface heating. Simulations are performed for a clear summer day at a latitude of $33^{\circ} \mathrm{N}$ and the differential thermal forcing in the building canyon is parameterized through a new potentially universal dimensionless number. In order to examine the validity of numerical modeling in representing the flow, performance of several turbulence models are compared with the wind tunnel experiment by Meinders [59]. Similar to Xie and Castro [70], this study concludes that Large Eddy Simulations (LES) performs well in resolving turbulent fluctuations in and above the urban-like obstacles. Reynolds-averaged Navier-Stokes (RANS) and Detached Eddy Simulation (DES) models are however shown to be inadequate, especially in representing the turbulent parameters within the canopy. Additionally, the correction of common but non-physical accumulation of heat in the periodic boundary conditions is performed by a constant inflow temperature boundary condition. Achieving a desired air setpoint temperature in the domain while allowing the flow and thermal field 

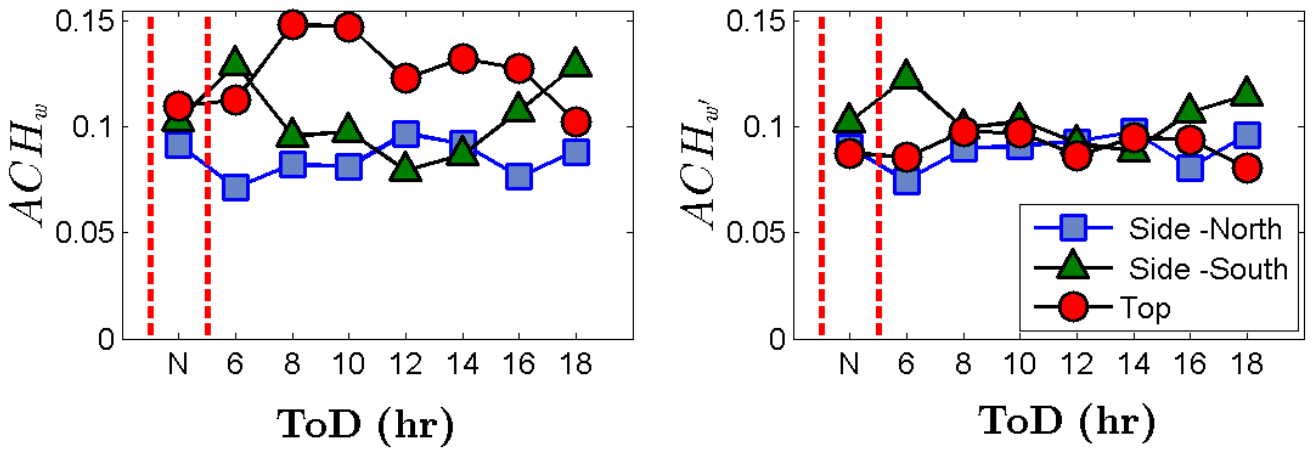

Figure 18: Air Exchange Rate at different ventilation surfaces of the building canyon as a function of ToD. Left: Total time-averaged ACH. Right: Time-averaged ACH induced by velocity fluctuations. The neutral case (N) is shown as a reference and separated with red dashed lines.

to evolve dynamically requires further research; a possible solution could be to update the heat source/sink term at the top boundary dynamically according to the surface heat flux.

The significance of considering realistic boundary conditions is emphasized in this paper. In summary, two factors influence the flow under realistic conditions: A) the three-dimensional geometry of the building canyon as opposed to 2D canyons with infinitely long width in the spanwise direction: The temperature gradient and therefore air exchange in the spanwise direction is shown to modify the canyon vortex significantly. For example, for a canyon aspect ratio of one, the center of the canyon vortex is above $z / H=0.75$ for our 3D simulations throughout the day, while reported to be at the center height for the 2-D canyon with the same aspect ration $(\mathrm{AR}=1)$. Additionally, the formation of the secondary vortex in the windward heating cases is not observed, which can also be attributed to flow forcing in the spanwise direction. B) the consideration of three-dimensional thermal forcing in the span-wise and stream-wise canyon: Walls and ground surfaces undergo different patterns of shaded and sunlit areas during the day, therefore more than one urban surfaces is often heated. In simplified uniform heating scenarios studied previously, the effect of multiple surface heating as well as heating in the streamwise canyon are often neglected, while we show that spatial patterns of surface heating generate horizontal temperature and pressure gradients in and above the canyon, influencing the strength of the canyon vortex. For example, canyon ground heating has received wide attention in wind tunnel experiments (such as Allegrini et al [22] and Uehara et al [11]) and numerical modeling (such as Kim and Baik [17] and $\mathrm{Li}$ et al [20]). In these papers, ground heating was shown to increase the strength of the main canyon vortex for relatively small aspect ratios $(A R=1-1.2)$. However, in realistic conditions, roof heating is present with ground heating and we observe a decrease of vortex strength instead, presumably because of horizontal temperature gradients at the roof level. Similarly, the variation of air exchange rate at different ventilating faces demonstrate the significance of combined heating of windward or leeward walls, with roof and north-south walls heating.

Richardson number has been applied frequently in categorizing the vortex structure inside the canyon with aspect to atmospheric stability and vertical wind shear. In addition to the vertical stratification, the horizontal temperature gradient creates buoyant forcing in the street canyon. Therefore buoyancy effect arising from ground surface heating and the resulting Richardson number are insufficient to describe atmospheric instability. Additionally, the geometry of the roughness was shown to influence the vortex formation and the relative importance of inertia to buoyancy forcing. Therefore, a second buoyancy parameter $\left(R i_{h}\right)$ is introduced that quantifies the orientation of wall heating with respect to the wind, and the horizontal temperature difference inside the canyon. while the canyon aspect ratio appears in the definition of $R i_{h}$ the effect of geometry is not studied in the present paper, and an analysis of the related similarity is left for future work. Also, the flow structure in a vertical plane through the canyon center is not significantly affected by leeward versus windward surface heating. 
Street canyon ventilation characterizes the amount of air entering or leaving the building canyon which has implications for air quality, especially dispersion of automobile emissions. Strong roof heating at 1200 PST decrease the total $\mathrm{ACH}$, while wall heating enhances the air removal for leeward and windward heating (in order of importance). Velocity fluctuations at ventilating faces is shown to be the primary mean of air removal, indicating the dominant effect of turbulence on ventilation from the street canyon. Under threedimensional thermal forcing, air exchange through the spanwise direction is significant (compared to roof ventilation) and exhibits asymmetrical behavior in north and south faces with ToD.

Air exchange from the street canyon is shown to be a function of horizontal temperature gradient. Therefore in a non-idealized configuration of buildings, the orientation of the street canyon can significantly affect the air exchange during daytime. For the range of Richardson numbers studied here (small vertical stratification since large roof heating and high wind speed counteract floor heating, and large horizontal variability during the day) the ACH is enhanced during periods when wall heating dominates, suggesting the north-south canyons would feature enhanced removal of pollutant.

\section{Acknowledgments}

We acknowledge funding from a National Science Foundation CAREER award.

\section{REFERENCES}

[1] Gerhard K Heilig. World urbanization prospects: the 2011 revision. United Nations, Department of Economic and Social Affairs (DESA), Population Division, Population Estimates and Projections Section, New York, 2012.

[2] Helmut E Landsberg. The urban climate, volume 28. Academic Press, 1981.

[3] Timothy R Oke. Review of urban climatology, 1968-1973. 1974.

[4] Timothy R Oke. Review of urban climatology, 1973-1976. 1979.

[5] Timothy R Oke. Boundary layer climates. Routledge, 2002.

[6] Haider Taha. Urban climates and heat islands: albedo, evapotranspiration, and anthropogenic heat. Energy and Buildings, 25(2):99-103, 1997.

[7] Yasuharu Nakamura and Timothy R Oke. Wind, temperature and stability conditions in an east-west oriented urban canyon. Atmospheric Environment (1967), 22(12):2691-2700, 1988.

[8] Jean-François Sini, Sandrine Anquetin, and Patrice G Mestayer. Pollutant dispersion and thermal effects in urban street canyons. Atmospheric Environment, 30(15):2659-2677, 1996.

[9] Yasushi Sakakibara. A numerical study of the effect of urban geometry upon the surface energy budget. Atmospheric Environment, 30(3):487-496, 1996.

[10] Jae-Jin Kim and Jong-Jin Baik. A numerical study of thermal effects on flow and pollutant dispersion in urban street canyons. Journal of Applied Meteorology, 38(9):1249-1261, 1999.

[11] Kiyoshi Uehara, Shuzo Murakami, Susumu Oikawa, and Shinji Wakamatsu. Wind tunnel experiments on how thermal stratification affects flow in and above urban street canyons. Atmospheric Environment, 34(10):1553-1562, 2000.

[12] Rizwan Ahmed Memon, Dennis YC Leung, and Chun-Ho Liu. Effects of building aspect ratio and wind speed on air temperatures in urban-like street canyons. Building and Environment, 45(1):176-188, 2010.

[13] Neda Yaghoobian, Jan Kleissl, and E Scott Krayenhoff. Modeling the thermal effects of artificial turf on the urban environment. Journal of Applied Meteorology and Climatology, 49(3):332-345, 2010.

[14] Neda Yaghoobian and Jan Kleissl. Effect of reflective pavements on building energy use. Urban Climate, 2:25-42, 2012.

[15] Negin Nazarian and Jan Kleissl. CFD simulation of an idealized urban environment: Thermal effects of geometrical characteristics and surface materials. Urban Climate, 12:141-159, 2015.

[16] Jose Luis Santiago, Omduth Coceal, and Alberto Martilli. How to parametrize urban-canopy drag to reproduce winddirection effects within the canopy. Boundary-layer Meteorology, 149(1):43-63, 2013.

[17] Jae-Jin Kim and Jong-Jin Baik. Urban street-canyon flows with bottom heating. Atmospheric Environment, 35(20):3395$3404,2001$.

[18] Bojan Ničeno, ADT Dronkers, and Kemal Hanjalić. Turbulent heat transfer from a multi-layered wall-mounted cube matrix: a large eddy simulation. International journal of heat and fluid flow, 23(2):173-185, 2002.

[19] A Kovar-Panskus, L Moulinneuf, E Savory, A Abdelqari, J-F Sini, J-M Rosant, A Robins, and N Toy. A wind tunnel investigation of the influence of solar-induced wall-heating on the flow regime within a simulated urban street canyon. Water, Air and Soil Pollution: Focus, 2(5-6):555-571, 2002.

[20] Xian-Xiang Li, Rex E Britter, Leslie K Norford, Tieh-Yong Koh, and Dara Entekhabi. Flow and pollutant transport in urban street canyons of different aspect ratios with ground heating: Large-eddy simulation. Boundary-Layer Meteorology, $142(2): 289-304,2012$. 
[21] Seung-Bu Park, Jong-Jin Baik, Siegfried Raasch, and Marcus Oliver Letzel. A large-eddy simulation study of thermal effects on turbulent flow and dispersion in and above a street canyon. Journal of Applied Meteorology and Climatology, $51(5): 829-841,2012$

[22] Jonas Allegrini, Viktor Dorer, and Jan Carmeliet. Wind tunnel measurements of buoyant flows in street canyons. Building and Environment, 59:315-326, 2013.

[23] Roland B Stull. An introduction to boundary layer meteorology, volume 13. Springer, 1988.

[24] Neda Yaghoobian and Jan Kleissl. An improved three-dimensional simulation of the diurnally varying street-canyon flow. Boundary-Layer Meteorology, 153(2):251-276, 2014.

[25] N Ito. Field experiment study on the convective heat transfer coefficient on exterior surface of a building. ASHRAE Trans.;(United States), 78, 1972.

[26] Steve Sharples. Full-scale measurements of convective energy losses from exterior building surfaces. Building and Environment, 19(1):31-39, 1984.

[27] Dennis Loveday and Ahmad Taki. Convective heat transfer coefficients at a plane surface on a full-scale building facade. International Journal of Heat and Mass Transfer, 39(8):1729-1742, 1996.

[28] Aya Hagishima and Jun Tanimoto. Field measurements for estimating the convective heat transfer coefficient at building surfaces. Building and Environment, 38(7):873-881, 2003.

[29] Y Liu and Douglas John Harris. Full-scale measurements of convective coefficient on external surface of a low-rise building in sheltered conditions. Building and Environment, 42(7):2718-2736, 2007.

[30] Anders Nottrott, Shiho Onomura, Atsushi Inagaki, Manabu Kanda, and Jan Kleissl. Convective heat transfer on leeward building walls in an urban environment: measurements in an outdoor scale model. International Journal of Heat and Mass Transfer, 54(15):3128-3138, 2011.

[31] Hajime Nakamura, Tamotsu Igarashi, and Takayuki Tsutsui. Local heat transfer around a wall-mounted cube in the turbulent boundary layer. International Journal of Heat and Mass Transfer, 44(18):3385-3395, 2001.

[32] Aya Hagishima, Jun Tanimoto, and Ken-ich Narita. Intercomparisons of experimental convective heat transfer coefficients and mass transfer coefficients of urban surfaces. Boundary-Layer Meteorology, 117(3):551-576, 2005.

[33] John Palyvos. A survey of wind convection coefficient correlations for building envelope energy systems' modeling. Applied Thermal Engineering, 28(8):801-808, 2008.

[34] Thijs Defraeye, Bert Blocken, and Jan Carmeliet. Convective heat transfer coefficients for exterior building surfaces: Existing correlations and CFD modelling. Energy Conversion and Management, 52(1):512-522, 2011.

[35] Marcelo G Emmel, Marc O Abadie, and Nathan Mendes. New external convective heat transfer coefficient correlations for isolated low-rise buildings. Energy and Buildings, 39(3):335-342, 2007.

[36] Bert Blocken, Thijs Defraeye, Dominique Derome, and Jan Carmeliet. High-resolution CFD simulations for forced convective heat transfer coefficients at the facade of a low-rise building. Building and Environment, 44(12):2396-2412, 2009.

[37] Thijs Defraeye, Bert Blocken, and Jan Carmeliet. CFD analysis of convective heat transfer at the surfaces of a cube immersed in a turbulent boundary layer. International Journal of Heat and Mass Transfer, 53(1):297-308, 2010.

[38] Habibollah Heidarzadeh, Mousa Farhadi, and Kurosh Sedighi. Convective heat transfer over a wall mounted cube using large eddy simulation. CFD Letters, 4(2):80-92, 2012.

[39] Hazim B Awbi. Calculation of convective heat transfer coefficients of room surfaces for natural convection. Energy and Buildings, 28(2):219-227, 1998.

[40] Ian Beausoleil-Morrison and Paul Strachan. On the significance of modelling internal surface convection in dynamic whole-building simulation programs. ASHRAE Transactions, 105(2):929-940, 1999.

[41] Ioannis Panagiotou, Marina K-A Neophytou, David Hamlyn, and Rex E Britter. City breathability as quantified by the exchange velocity and its spatial variation in real inhomogeneous urban geometries: An example from central london urban area. Science of the Total Environment, 442:466-477, 2013.

[42] Chun-Ho Liu and Mary C Barth. Large-eddy simulation of flow and scalar transport in a modeled street canyon. Journal of Applied Meteorology, 41(6):660-673, 2002.

[43] Chun-Ho Liu, Mary C Barth, and Dennis YC Leung. Large-eddy simulation of flow and pollutant transport in street canyons of different building-height-to-street-width ratios. Journal of Applied Meteorology, 43(10):1410-1424, 2004.

[44] Chun-Ho Liu, Dennis YC Leung, and Mary C Barth. On the prediction of air and pollutant exchange rates in street canyons of different aspect ratios using large-eddy simulation. Atmospheric Environment, 39(9):1567-1574, 2005.

[45] Xian-Xiang Li, Chun-Ho Liu, and Dennis YC Leung. Development of a k- $\varepsilon$ model for the determination of air exchange rates for street canyons. Atmospheric environment, 39(38):7285-7296, 2005.

[46] Xiaomin Xie, Chun-Ho Liu, Dennis YC Leung, and Michael KH Leung. Characteristics of air exchange in a street canyon with ground heating. Atmospheric Environment, 40(33):6396-6409, 2006.

[47] WC Cheng, Chun-Ho Liu, and Dennis YC Leung. On the correlation of air and pollutant exchange for street canyons in combined wind-buoyancy-driven flow. Atmospheric Environment, 43(24):3682-3690, 2009.

[48] Xian-Xiang Li, Chun-Ho Liu, and Dennis YC Leung. Numerical investigation of pollutant transport characteristics inside deep urban street canyons. Atmospheric Environment, 43(15):2410-2418, 2009.

[49] Jong-Jin Baik, Yoon-So Kang, and Jae-Jin Kim. Modeling reactive pollutant dispersion in an urban street canyon. Atmospheric Environment, 41(5):934-949, 2007.

[50] Y Toparlar, B Blocken, P Vos, GJF van Heijst, WD Janssen, T van Hooff, H Montazeri, and HJP Timmermans. Cfd simulation and validation of urban microclimate: A case study for bergpolder zuid, rotterdam. Building and Environment, 83:79-90, 2015.

[51] Hong Chen, Ryozo Ooka, Kazuya Harayama, Shinsuke Kato, and Xiaofeng Li. Study on outdoor thermal environment of apartment block in shenzhen, china with coupled simulation of convection, radiation and conduction. Energy and 
Buildings, 36(12):1247-1258, 2004.

[52] Rajagopalan Priyadarsini, Wong Nyuk Hien, and Cheong Kok Wai David. Microclimatic modeling of the urban thermal environment of singapore to mitigate urban heat island. Solar energy, 82(8):727-745, 2008.

[53] Xiaoshan Yang, Lihua Zhao, Michael Bruse, and Qinglin Meng. Evaluation of a microclimate model for predicting the thermal behavior of different ground surfaces. Building and Environment, 60:93-104, 2013.

[54] Ian D Stewart and Tim R Oke. Local climate zones for urban temperature studies. Bulletin of the American Meteorological Society, 93(12):1879-1900, 2012.

[55] O Coceal, TG Thomas, IP Castro, and SE Belcher. Mean flow and turbulence statistics over groups of urban-like cubical obstacles. Boundary-Layer Meteorology, 121(3):491-519, 2006.

[56] EH Chui and GD Raithby. Computation of radiant heat transfer on a nonorthogonal mesh using the finite-volume method. Numerical Heat Transfer, 23(3):269-288, 1993.

[57] Ansys Fluent. 12.0 theory guide. Ansys Inc, 5, 2009.

[58] H Werner and H Wengle. Large-eddy simulation of turbulent flow over and around a cube in a plate channel. In Turbulent Shear Flows 8, pages 155-168. Springer, 1993.

[59] Erwin Rinaldo Meinders. Experimental study of heat transfer in turbulent flows over wall-mounted cubes. PhD thesis, Delft University of Technology, 1998.

[60] Massimo Germano, Ugo Piomelli, Parviz Moin, and William H Cabot. A dynamic subgrid-scale eddy viscosity model. Physics of Fluids A: Fluid Dynamics (1989-1993), 3(7):1760-1765, 1991.

[61] Mikhail L Shur, Philippe R Spalart, Mikhail Kh Strelets, and Andrey K Travin. A hybrid RANS-LES approach with delayed-des and wall-modelled LES capabilities. International Journal of Heat and Fluid Flow, 29(6):1638-1649, 2008.

[62] Philippe R Spalart, S Deck, ML Shur, KD Squires, M Kh Strelets, and A Travin. A new version of detached-eddy simulation, resistant to ambiguous grid densities. Theoretical and Computational Fluid Dynamics, 20(3):181-195, 2006.

[63] JL Santiago, ES Krayenhoff, and A Martilli. Flow simulations for simplified urban configurations with microscale distributions of surface thermal forcing. Urban Climate, 9:115-133, 2014.

[64] Harindra JS Fernando, Dragan Zajic, Silvana Di Sabatino, Reneta Dimitrova, Brent Hedquist, and Ann Dallman. Flow, turbulence, and pollutant dispersion in urban atmospheres. Physics of Fluids, 22(5):051301, 2010.

[65] Ann Dallman, Sigurdur Magnusson, Rex E Britter, Leslie K Norford, Dara Entekhabi, and Harindra JS Fernando. Conditions for thermal circulation in urban street canyons. Building and Environment, 2014.

[66] Sigurdur Magnusson, Ann Dallman, Dara Entekhabi, Rex E Britter, Harindra JS Fernando, and Leslie K Norford. On thermally forced flows in urban street canyons. Environmental Fluid Mechanics, pages 1-15, 2014.

[67] Xiaoming Cai. Effects of differential wall heating in street canyons on dispersion and ventilation characteristics of a passive scalar. Atmospheric Environment, 51:268-277, 2012.

[68] Brian Offerle, Ingegärd Eliasson, CSB Grimmond, and Björn Holmer. Surface heating in relation to air temperature, wind and turbulence in an urban street canyon. Boundary-layer Meteorology, 122(2):273-292, 2007.

[69] Long Sun, Anders Nottrott, and Jan Kleissl. Effect of hilly urban morphology on dispersion in the urban boundary layer. Building and Environment, 48:195-205, 2012

[70] Zheng-Tong Xie and Ian P Castro. Large-eddy simulation for flow and dispersion in urban streets. Atmospheric Environment, 43(13):2174-2185, 2009.

[71] ASHRAE Handbook. Fundamentals. American Society of Heating, Refrigerating and Air Conditioning Engineers, Atlanta, 111, 2001.

[72] Stephen B Pope. Turbulent flows. Cambridge University Press, 2000.

[73] Joseph Smagorinsky. General circulation experiments with the primitive equations: I. the basic experiment*. Monthly Weather Review, 91(3):99-164, 1963.

[74] Ugo Piomelli, Joel H Freziger, and Parviz Moin. Model consistency in large eddy simulation of turbulent channel flows. Physics of Fluids, 31(7):1884, 1988.

[75] PR Spalart, WH Jou, M Strelets, and SR Allmaras. Comments on the feasibility of LES for wings, and on a hybrid RANS/LES approach. Advances in DNS/LES, 1:4-8, 1997.

[76] Phillipe R Spalart and Steven R Allmaras. A one-equation turbulence model for aerodynamic flows. 1992.

[77] M Shur, PR Spalart, M Strelets, and A Travin. Detached-eddy simulation of an airfoil at high angle of attack. Engineering Turbulence Modelling and Experiments, 4:669-678, 1999.

[78] Tsan-Hsing Shih, William W Liou, Aamir Shabbir, Zhigang Yang, and Jiang Zhu. A new k-epsilon eddy viscosity model for high reynolds number turbulent flows. Computers and Fluids, 24(3):227-238, 1995. 


\section{APPENDICES}

\section{APPENDIX A - Detailed Model Description}

Turbulence modeling and heat transfer calculations are performed using the unstructured finite volume solver ANSYS FLUENT 14.5. The domain configuration is described in Section 2. The soil layer depth is chosen to be several diurnal thermal damping depths and material properties of ground and wall surfaces are as in Table 3. Since wall and roof thermal thicknesses are small relative to the ground, they are not resolved by the grid; instead the 1D steady heat conduction equation is used to calculate the external surface temperature. The temperature boundary conditions inside the buildings and at the base of the deepest soil sublayer (exterior surfaces of computational domain) are set to be constant (295 K). To maintain a realistic air temperature and correct for accumulation of heat due to large convective heat transfer and the periodic boundary condition, the homogeneous temperature at the inlet Ta is reset to follow the TMY3 diurnal cycle. An effort was made to set a more realistic temperature profile at the inlet with an average vertical temperature profile of $\mathrm{Ta}(\mathrm{z})$, however with periodic boundary conditions FLUENT only permits homogeneous temperatures at the inlet. Temperature is only fixed to the inlet air temperature at the very first grid point in the stream-wise direction $\left(T_{a}(j x=1, j y, j z)\right)$. Apart from that node, the temperature profile evolves dynamically in the computational domain. The boundary condition for temperature at the top boundary is set to zero gradient.

Table 3: Thermal and radiative properties of building walls, roof and ground sublayers. For wall and rood surfaces, effective thermal conductivity and heat capacity are calculated based on multi-layered material properties described in Yaghoobian and Kleissl $[13]$.

\begin{tabular}{|c|c|c|c|c|c|c|}
\hline & & $\begin{array}{c}\text { Thickness } \\
\text { (m) }\end{array}$ & $\begin{array}{c}\text { Effective } \\
\text { thermal } \\
\text { conductivity } \\
\left(\mathrm{W} \mathrm{m}^{-1} \mathrm{~K}^{-1}\right)\end{array}$ & - & - & $\begin{array}{l}\text { Effective heat } \\
\text { capacity } \\
\left(\mathrm{MJ} \mathrm{m}^{-3} \mathrm{~K}^{-1}\right)\end{array}$ \\
\hline Roof & & 0.234 & 0.043 & 0.92 & 0.15 & 0.182 \\
\hline wall & & 0.113 & 0.045 & 0.88 & 0.30 & 0.187 \\
\hline \multicolumn{7}{|c|}{ Ground } \\
\hline & layer 1 & 0.070 & 0.75 & 0.95 & 0.18 & 1.940 \\
\hline & layer 2 & 0.700 & 0.95 & & & 2.100 \\
\hline & layer 3 & 1.600 & 0.87 & & & 2.700 \\
\hline
\end{tabular}

Reynolds' analogy between momentum and heat transport and the wall function by Werner and Wangle [58] are used to calculate the surface convective heat fluxes and Convective Heat Transfer Coefficients $(\mathrm{CHTCs})^{1}$. The surface energy balance consisting of longwave $(L)$ and shortwave $(S)$ radiation, conduction $\left(Q_{c}\right)$ and convection $\left(Q_{h}\right)$ heat flux is as Eq 9-11 and latent heat flux is neglected.

$$
\begin{array}{r}
(1-\alpha) S \downarrow-L=Q_{c}+Q_{h} \\
L=\epsilon\left[\sigma T^{4}-L_{i n c}\right] \\
L_{i n c, g r}=\left(F_{s k y-g r}\right) \sigma T_{s k y}^{4}+L_{w-g r}
\end{array}
$$

Solar radiation was not taken from TMY3 but rather calculated using the ANSYS Solar Load Model for June 21st and solar noon was approximately at 1150 LST. The direct solar irradiance ranges from 0 at night

\footnotetext{
${ }^{1}$ Since FLUENT does not output the convective fluxes explicitly, they were obtained from Eq 9 and net shortwave, longwave, and conduction heat flux as output by FLUENT.
} 
to approximately $900 \mathrm{~W}$ m-2 at solar noon. The solar ray tracing algorithm (SRTA) takes a beam of direct radiation in the direction of the sun position vector, applies it to opaque surfaces and performs a face-by-face shading analysis to determine shadows. The total reflected solar irradiation is distributed among all surfaces weighted by area depending on the scattering fraction (set to 1 in this study). The Solar Load Model also accounts for isotropic diffuse radiation based on the approach suggested in the 2001 ASHRAE Fundamentals Handbook [71] (Chapter 20, Fenestration).

All computations are performed with the pressure-based solver, PRESTO! for pressure discretization, second order upwind schemes for Momentum, Energy and Discrete Ordinate Equations and the PISO scheme for pressure-velocity coupling. First order implicit scheme is used for time discretization (see [57] and the references cited therein for more details on these schemes). The time marching is performed using a 2 nd order implicit transient formulation.

\section{APPENDIX B - Large Eddy Simulation}

In LES the larger three dimensional unsteady turbulent motions are directly represented, whereas the effect of small scales of motion is modeled [72]. By applying a filtering operation, velocity is decomposed into the sum of a resolved (filtered) and a residual components i.e, $u=\hat{u}+u^{\prime}$ where $\hat{()}$ is a filtered quantity. The nonlinear term $\widehat{u_{i} u_{j}}$ appears in the non-dimensional incompressible form of Navier-Stokes equations and needs to be modeled. It is common to rewrite this term with the subgrid-scale stress $\left(\tau_{i j}\right)$ and split the additional stresses into an anisotropic part and add the isotropic part to the pressure. The resulting LES equations are

$$
\begin{aligned}
& \frac{\partial \hat{u}_{i}}{\partial x_{i}}=0, \\
& \frac{\partial}{\partial t}\left(\hat{u}_{i}\right)+\frac{\partial}{\partial x_{i}}\left(\hat{u}_{i} \hat{u}_{j}\right)=-\frac{1}{\rho} \frac{\partial P^{*}}{\partial x_{i}}-\frac{\partial \tau_{i j}}{\partial x_{j}}+\frac{\partial}{\partial x_{j}}\left[\nu\left(\frac{\partial \hat{u}_{i}}{\partial x_{j}}+\frac{\partial \hat{u}_{j}}{\partial x_{i}}\right)\right] .
\end{aligned}
$$

Filtering the energy equation, one obtains

$$
\frac{\partial \rho \hat{h_{s}}}{\partial t}+\frac{\partial \rho \hat{u_{i}} \hat{h_{s}}}{\partial x_{i}}-\frac{\partial \hat{p}}{\partial t}-\hat{u_{j}} \frac{\partial \hat{p}}{\partial x_{i}}-\frac{\partial}{\partial x_{i}}\left(\lambda \frac{\partial \hat{T}}{\partial x_{i}}\right)=-\frac{\partial}{\partial x_{j}}[\underbrace{\rho\left(\widehat{u_{i} h_{s}}-\hat{u}_{i} \hat{h_{s}}\right)}_{\text {subgrid enthalpy flux }}]
$$

where $h_{s}$ and $\lambda$ are the sensible enthalpy and thermal conductivity, respectively. The subgrid enthalpy flux term in the equation above is approximated using the gradient hypothesis:

$$
\rho\left(\widehat{u_{i} h_{s}}-\hat{u_{i}} \hat{h_{s}}\right)=-\frac{\mu_{\mathrm{SGS}} C_{p}}{\operatorname{Pr}_{\mathrm{SGS}}} \frac{\partial \hat{T}}{\partial \hat{x}_{j}},
$$

where $\mu_{\mathrm{SGS}}$ is a subgrid scale viscosity, and $\operatorname{Pr}_{\mathrm{SGS}}$ is the subgrid scale Prandtl number equal to 0.85. More details of the Subgrid Scale models implementation in ANSYS FLUENT and their validations can be found in $[57]$.

\section{APPENDIX C - Algebraic Wall-Modeled LES Model (WMLES)}

The energy containing scales in the turbulent spectrum are small near the wall and require an excessively high resolution and a small time step for wall-resolving LES models. The Reynolds number scaling limitations of LES can be overcome using the Algebraic Wall-Modeled LES (WMLES) approach [61]. In WMLES, RANS is applied for wall-distances $0<d_{w}<0.5 h_{\max }$, where $d_{w}$ is the distance to the wall and $h_{\max }$ is the maximum local grid spacing. With the increase in wall distance an empirical blending function provides rapid switching of the model from RANS to LES for $0.5 h_{\max }<d_{w}<h_{\max }$. 
The Algebraic WMLES formulation proposed by Shur et.al. [61] combines a mixing length model with a modified Smagorinsky model [73] and with the wall damping function of [74]. The eddy viscosity is calculated with the use of a hybrid length scale:

$$
v_{t}=\min \left[\left(k d_{w}\right)^{2},\left(C_{s} \Delta\right)^{2}\right]|\hat{S}|\left[1-\exp \left[-\left(\frac{y^{+}}{25}\right)^{3}\right]\right]
$$

where $|\hat{S}|$ is the magnitude of the strain rate tensor, $k=0.41$ is the van Karman constant, $C_{s}=0.2$ is the Smagorinsky constant, and $y^{+}$is the normal to the wall inner scaling. The WMLES model is based on a modified grid scale to account for the grid anisotropies in wall-modeled flows:

$$
\Delta=\min \left(\max \left(C_{w} d_{w} ; C_{w} h_{\max } ; h_{w n}\right), h_{\max }\right) .
$$

Here $h_{\max }$ is the maximum edge length of the cell, $h_{w n}$ is the wall-normal grid spacing, and $C_{w}=0.15$ is a constant.

\section{APPENDIX D - Detached Eddy Simulation}

This section describes the two Delayed Detached Eddy Simulation (DDES) models that are compared with experimental results and LES model in section 3. DES was originally proposed by Spalart et al. [75] to address high Reynolds number flows over complex wall-bounded configurations using affordable computational resources. In the DES approach, the unsteady RANS models are employed in the boundary layer, while the subgrid scale formulation of LES is applied in the regions where the resolution is fine enough for LES. The LES region covers the turbulent region where large unsteady eddies play a dominant role. In the near-wall region, the respective RANS models are recovered.

\section{- Spalart-Allmaras based DDES model}

The standard Spalart-Allmaras model uses the distance to the closest wall as the definition for the length scale $d$, which plays a major role in determining the level of turbulent production or destruction [76]. The DES model, as proposed by Shur et al. [77] replaces $d$ everywhere with a new length scale $\tilde{d}$, defined as

$$
\tilde{d}=\min \left(d, C_{D E S} \Delta_{\max }\right)
$$

where the grid spacing $\Delta_{\max }$ is the largest grid spacing in the $x, y$, or $z$ directions. The empirical constant $C_{d e s}$ has a value of 0.65 . Accordingly, where the grid is not fine enough to sustain resolved turbulence, the LES mode is activated inside the boundary layer. Delayed detached-eddy simulation (DDES) by Spalart et al [62] is further introduced to preserve the RANS mode throughout the boundary layer and $\tilde{d}$ is re-defined according to:

$$
\tilde{d}=d-f_{d} \min \left(0, \mathrm{~d}-\mathrm{C}_{\mathrm{des}} \Delta_{\max }\right)
$$

where $f_{d}$ is given by:

$$
f_{d}=1-\tanh \left(\left(8 r_{d}\right)^{3}\right)
$$

and

$$
r_{d}=\frac{\nu_{t}+\nu}{\sqrt{U_{i, j} U_{i, j}} k^{2} d} ; k=0.41
$$

- Realizable $k-\epsilon$ based DDES model 
The Realizable $k-\epsilon$ based DES model is similar to Realizable $k-\epsilon$ RANS model by Shih et al [78] with the exception of the dissipation term in the $k$ equation. In the DES model, the realizable $k-\epsilon$ RANS dissipation term is modified such that

$$
Y_{k}=\frac{\rho k^{\frac{3}{2}}}{l_{d e s}},
$$

where

$$
\begin{array}{r}
l_{\text {des }}=\min \left(l_{\text {rke }}, l_{\text {les }}\right) \\
l_{\text {rke }}=\frac{k^{\frac{3}{2}}}{\epsilon} \\
l_{\text {les }}=C_{\text {des }} \Delta_{\max },
\end{array}
$$

where $C_{d e s}$ is a calibration constant used in the DES model and has a value of 0.61. Similarly to the SpalartAllmaras model, the delayed concept can be applied as well to the Realizable DES model to preserve the RANS mode throughout the boundary layer. The DES length $l_{\text {des }}$ is redefined such that

$$
l_{\text {des }}=l_{r k e}-f_{d} \max \left(0, l_{r k e}-C_{d e s} \Delta_{\max }\right) .
$$

